\title{
Femmes salariées et non salariées : quelles différences de temps de travail?
}

Self-Employed and Salaried Women: What Working Time Differences?

Amandine Barrois et François-Xavier Devetter

\section{(2) OpenEdition}

Édition électronique

URL : http://journals.openedition.org/travailemploi/7543

DOI : 10.4000/travailemploi.7543

ISSN : 1775-416X

Éditeur

DARES - Ministère du Travail

\section{Édition imprimée}

Date de publication : 1 avril 2017

Pagination : 101-130

ISSN : 0224-4365

\section{Référence électronique}

Amandine Barrois et François-Xavier Devetter, « Femmes salariées et non salariées : quelles

différences de temps de travail ? », Travail et Emploi [En ligne], 150 | avril-juin 2017, mis en ligne le 11 juillet 2019, consulté le 05 septembre 2019. URL : http://journals.openedition.org/travailemploi/7543 ; DOI : 10.4000/travailemploi.7543 


\title{
Femmes salariées et non salariées : quelles différences de temps de travail ?*
}

\author{
Amandine Barrois ${ }^{* *}$, François-Xavier Devetter ${ }^{* * *}$
}

\begin{abstract}
L'objectif de cet article est de mieux comprendre, à partir d'une analyse de l'enquête Conditions de travail 2013, les particularités des temps de travail des femmes non salariées. Bien que cette catégorie regroupe des situations très diverses, comparée à celle des salariées, elle se révèle en moyenne marquée par des durées quotidiennes et hebdomadaires longues, des horaires atypiques et imprévisibles. La disponibilité temporelle au travail des femmes non salariées est donc forte. Cette réalité apparaît paradoxale face aux discours mettant en exergue la souplesse et les possibilités de conciliation des temps personnels et professionnels que ce statut est censé permettre. Trois groupes sont distingués en fonction du niveau du diplôme le plus élevé obtenu. Si les contraintes temporelles sont importantes pour l'ensemble des non-salariées, les résultats soulignent également la position particulière des moins qualifiées. Elles cumulent horaires importants et faibles rémunérations, posant ainsi la question des frontières entre travail domestique et travail non salarié.
\end{abstract}

$\mathrm{L}$ es économies européennes du $\mathrm{XX}^{\mathrm{e}}$ siècle ont connu une extension continue du salariat : depuis les années 1950, la part du travail indépendant dans l'emploi total a considérablement diminué en France, comme dans les autres pays de l'Organisation de coopération et de développement économiques (OCDE), en raison de la baisse du nombre d'exploitants agricoles et de la salarisation du secteur, ainsi que de la restructuration de certaines activités tertiaires. L'activité économique indépendante est ainsi passée du tiers de l'emploi total dans les années 1950 à moins de $10 \%$ à la fin des années 1990 (MARCHAND, ThÉLOT, 1997 ; COE, 2014). Ce déclin quantitatif peut expliquer que cette catégorie ait moins retenu l'attention des chercheurs. Elle apparaît en effet largement définie « par défaut », en référence au salariat : les non-salariés, selon

\footnotetext{
* Ce travail a été réalisé dans le cadre du projet CLEAN-ANR-13-JSH1-0001-01.

** Centre lillois d'études et de recherches sociologiques et économiques (Clersé), université Lille 1 ; amandine_barrois@live.fr.

*** Clersé, IMT Lille Douai ; francois-xavier.devetter@imt-lille-douai.fr.
} 
l'Institut national de la statistique et des études économiques (Insee), « se distinguent des salariés par l'absence de contrat de travail et par le fait qu'ils n'ont pas de lien de subordination juridique permanente à l'égard d'un donneur d'ordre ${ }^{1}{ }^{\prime}$. L'activité indépendante est donc considérée comme « le complémentaire, dans l'emploi total, du salariat, retenu comme catégorie centrale de l'analyse statistique de l'emploi et du droit du travail » (LuRTON, TOUTLEMONDE, 2007, p. 4).

Pourtant, plus récemment, face à la progression du chômage, des formes particulières d'emploi et du sous-emploi, le travail indépendant a suscité un intérêt croissant, du fait de sa possible capacité à créer des emplois (ARUM, Muller, 2004). Sous l'impulsion de la stratégie européenne pour l'emploi, la France a « incité à la création de petites entreprises et [favorisé] l'orientation des demandeurs d'emploi vers ces structures productives de taille réduite [en améliorant] l'environnement économique, fiscal, administratif et social des petites entreprises » (RAPELLI, LeSPAGNOL, 2007, p. 1). Portée par différentes mesures de simplification des formalités de création, de soutien financier, ainsi que par l'instauration en 2009 du régime de l'auto-entrepreneur, la part de l'activité indépendante est légèrement remontée. Ainsi, en 2013, ce sont 2,9 millions de personnes qui travaillent en tant que non salarié.e.s, soit 11,2\% des personnes en emploi (GugGEMOS, VIDALENC, 2014). Plus encore, le travail indépendant a retrouvé une forme d'attractivité symbolique : moins associé aux faibles protections, ce statut véhicule également une image positive de liberté et d'autonomie. Les discours positifs sur l'abandon des contraintes du salariat en faveur de l'autonomie permise par l'autoemploi se sont ainsi largement développés dans les médias. Ils concernent notamment les femmes ou les mères de famille (voir par exemple D'ANDRIA, 2014 ; Nel et al., 2010 ; BотеZ, 2010). Ce nouvel essor du travail indépendant a provoqué un intérêt croissant sur le plan académique et les recherches portant sur le non-salariat se sont multipliées (par exemple InSERM, 2011 ; CÉLÉRIER, 2014 ; OMALEK, RIOUX, 2015).

Cependant, certains aspects de cette évolution demeurent assez méconnus, dont la situation spécifique des femmes non salariées. Sur la période récente, l'analyse des inégalités de genre s'est le plus souvent concentrée sur les salariées - à de rares exceptions près, comme l'étude de Florent FAVRE (2009) centrée sur les revenus ou l'article de Susanne BURRI (2010) sur les protections juridiques. En statistique économique et sociale, l'arbitrage entre salariat et travail indépendant est ainsi peu interrogé sous l'angle des différences selon le sexe et la population des femmes indépendantes demeure méconnue ${ }^{2}$.

Les transformations de l'emploi peuvent-elles redessiner un choix entre emploi salarié et travail indépendant et ce, plus spécifiquement pour les femmes ? Elles trouveraient dans le statut de travailleur indépendant une liberté de temps de travail facilitant de nouvelles formes de conciliation entre leurs vies professionnelle et familiale.

1. Définition en ligne sur le site de l'Insee : https://www.insee.fr/fr/metadonnees/definition/c1748, consulté le 13 octobre 2017.

2. Certains travaux ont cependant étudié les spécificités des femmes cheffes d'entreprises (DUCHÉNEAUT, ORHAN, 2000 ; INSEE, 2004 ; FOUQUET, 2005 ; cf. également CARRIER et al., 2006, pour une synthèse des travaux nord-américains). 
L'emploi non salarié peut cependant également apparaître pour nombre d'entre elles comme une solution subie faute d'emploi salarié disponible. L'objectif de cet article est de mieux comprendre la situation spécifique du temps de travail des femmes non salariées, à la fois en termes de durées, d'horaires sur la journée ou la semaine, et de leur maîtrise. En d'autres termes, le statut non salarié peut-il être un choix lié à une volonté de mieux contrôler son temps de travail et permet-il d'obtenir une meilleure rémunération de la disponibilité temporelle offerte ${ }^{3}$ ? Ou apparaît-il au contraire comme un statut dégradé au regard des temps de travail réalisés et des rémunérations obtenues ?

Pour apporter des éléments de réponse à ces questions, nous nous sommes appuyés sur la dernière édition (2013) de l'enquête Conditions de travail de la Direction de l'animation de la recherche, des études et des statistiques (Dares) (encadré). Dans un premier temps, nous soulignons l'hétérogénéité de la catégorie de travailleuses non salariées en distinguant notamment trois groupes en fonction du niveau de diplôme le plus élevé de celles qui occupent ces emplois. Dans un deuxième temps, nous cherchons à décrire les différentes dimensions (durées, horaires, prévisibilité) des temps de travail de ces trois groupes en les comparant à celles des femmes salariées ayant des niveaux de qualification équivalents. Enfin, la troisième partie nous permettra de souligner deux contraintes auxquelles sont confrontées les travailleuses non salariées de manière plus fréquente que les salariées : des difficultés fortes en termes de conciliations en dépit d'une grande liberté apparente de temps de travail, d'une part, et une exposition fréquente aux basses ou très basses rémunérations, d'autre part.

\section{ENCADRÉ \\ Source et méthode}

Pour cette recherche, nous nous sommes appuyés sur l'enquête Conditions de travail réalisée par la Dares, ministère du Travail, de l'Emploi, de la Formation professionnelle et du Dialogue social, pour l'année 2013. Reconduite tous les sept ou huit ans depuis 1978, cette enquête était menée en complément de l'enquête Emploi de l'Insee jusqu'en 2005. Pour l'édition 2013, elle a été conduite d'octobre 2012 à mars 2013, indépendamment de l'enquête Emploi, mais toujours par le réseau des enquêteurs de l'Insee afin de garantir une certaine continuité dans les modalités de passation. Son objectif est de fournir une description statistique des situations de travail, telles qu'elles sont vécues par le travailleur et non telles qu'elles sont prescrites par l'employeur. Les thèmes abordés ont été enrichis au fil des années pour tenir compte des évolutions de la réalité du travail, et concernent en 2013 : l'organisation du temps de travail ; les contraintes physiques ; la prévention et les accidents ; l'organisation et le rythme du travail ; la santé et les risques psychosociaux.

3. La disponibilité temporelle correspond à une vision large des temps de travail intégrant non seulement la durée hebdomadaire et quotidienne, mais également la localisation, la variabilité et la prévisibilité des horaires de travail (DEVETTER, 2001). 
Notre travail se base essentiellement sur les questions portant sur l'organisation du travail et du temps de travail. L'enquête concerne tous les actifs exerçant un emploi, qu'ils soient salariés ou non salariés, et est représentative de la population active occupée, âgée de 15 ans et plus. Pour l'année 2013, plus de 30000 individus ont été interrogés. Après avoir écarté les individus pour lesquels trop de valeurs étaient manquantes, nous avons pu conserver 27121 salarié.e.s et 3259 non-salariée.es ${ }^{1}$ (dont 1137 femmes). Il s'agit de travailleur·se·s indépendant·e·s ou à leur compte, de chef·fe·s d'entreprise salarié.e.s, de président·e·s directeur·rice·s généraux·ales, de gérant·e·s minoritaires ou associé·e·s.

Pour répondre aux questions que nous nous posons, nous utilisons une méthodologie quantitative en centrant la comparaison entre les femmes non salariées, d'une part, et les femmes salariées, d'autre part. La situation des hommes est parallèlement mobilisée en contrepoint.

D'un point de vue de méthode, il est important de souligner la dimension déclarative des données. Certaines variables peuvent avoir un sens un peu différent selon le statut des enquêté·e·s ou être recueillies selon des modalités légèrement distinctes. C'est notamment le cas des variables clés qui nous intéressent ici : le temps de travail et la rémunération. En effet, la notion de temps de travail que nous retenons ici est la durée habituellement travaillée, qui peut davantage varier pour les non-salarié·e·s, n'étant a priori pas influencée par une durée contractuelle (dans les faits comme dans les esprits). La fréquence particulièrement élevée pour les non-salarié·e·s de certaines durées (40 heures, 45 heures ou 50 heures) illustre un mécanisme de réponse qui ne s'appuie pas sur une durée contractuelle mais se réfère à des arrondis. De même, pour la variable mesurant les revenus mensuels, alors que les salarié·e·s sont amené·e·s à déclarer leur salaire net mensuel, les non-salarié·e·s déclarent leurs revenus annuels, qui sont ensuite divisés par douze. Dans les deux cas, un certain nombre de non-réponses ou de valeurs aberrantes réduisent l'échantillon : on dénombre 3131 répondant·e·s sur la durée du travail et 1891 sur les revenus ${ }^{2}$ du côté des non-salarié·e·s (contre 24577 répondant·e·s à ces deux variables pour les salarié·e·s). Les conclusions de certaines comparaisons, principalement sur les rémunérations, doivent donc demeurer prudentes. Le revenu mensuel moyen des non-salarié·e·s (2 587 euros) dans l'enquête est par exemple plus faible que celui obtenu par l'Insee via l'exploitation des sources administratives (3 260 euros) qui ne concerne cependant pas le champ agricole (SAlEMBIER, ThÉRON, 2016). Hors agriculture, les estimations issues des données de l'enquête se rapprochent davantage de celles résultant des données administratives, tout en restant plus faibles (2 953 euros).

1. Dans le fichier initial, on décompte 3475 travailleur·euse·s non salarié·e·s au sein desquel·le·s 210 observations ont été supprimées, dont un tiers correspond à des femmes.

2. La composition de l'échantillon en termes de sexe et de niveau de diplôme demeure toutefois quasiment inchangée : parmi les non-salarié·e·s, il y a $30 \%$ de femmes dans l'échantillon initial et $29 \%$ dans l'échantillon réduit (et respectivement $39,5 \%$ et $38,5 \%$ de peu diplômées). 


\section{Qui sont les femmes non salariées ?}

Construite en creux, par opposition au salariat, la catégorie de non-salariée est hétérogène (EVAIN, AMAR, 2006 ; BEFFY, 2006) et parfois difficile à délimiter. Nous revenons brièvement sur les principales lignes de fractures repérables en son sein, avant de distinguer trois groupes de travailleuses non salariées en fonction de leur niveau de diplôme.

\section{Les femmes non salariées : une catégorie hétérogène}

Plusieurs lignes de fractures peuvent être repérées au sein du monde des non-salarié·e·s (Amossé, Goux, 2004). Elles apparaissent au niveau détaillé de la nomenclature des professions et des catégories socioprofessionnelles, celui des professions, au-delà des grandes catégories qui regroupent la majorité des non-salariéee-s (DesRosières, THÉVENOT, 2002) : les exploitants agricoles, les commerçants, les artisans et les chefs d'entreprise, ainsi que les professions libérales.

En partie constitutif de ces catégories mais les traversant également, le statut juridique (être aide familial, indépendant ou employeur ; le cas échéant, le nombre de salariés), le secteur d'activité ou encore le niveau de diplôme sont des éléments centraux qui structurent la catégorie des non-salariés. Sur ces dimensions, la situation des femmes et des hommes est par ailleurs sensiblement différente (tableau 1).

Les femmes non salariées sont bien moins fréquemment employeuses que les hommes non salariés : c'est le cas de $28 \%$ d'entre elles, contre $43 \%$ des hommes. Dans la grande majorité des cas, les femmes non salariées ne sont donc pas employeuses. Et elles encadrent même moins souvent des salarié.e.s que la moyenne des femmes salariées : $27 \%$, contre $29 \%$ des femmes salariées (à titre de comparaison, $42 \%$ des hommes non salariés déclarent «encadrer des salariés »; estimation non reproduite dans le tableau). Enfin les relations commerciales qu'implique leur activité sont bien davantage tournées vers des particuliers que vers d'autres entreprises (56\%, contre $35 \%$ pour les hommes ${ }^{4}$ ).

Les non-salarié.e.s se démarquent ensuite des salarié.e.s selon le secteur d'activité auquel elles et ils appartiennent : le poids de l'agriculture demeure important tant pour les femmes que pour les hommes (une non-salariée sur six environ) alors que le secteur de la construction ne comprend que très peu de femmes à leur compte. Comme pour les salariées, le secteur des services regroupe une majorité de femmes non salariées, dont $31 \%$ travaillent dans la santé et $15 \%$ dans le commerce.

Il est important de souligner que les secteurs d'activité où se situent les nonsalarié.e.s sont sensiblement différents de ceux qui rassemblent les salarié.e.s. Comparer

4. Le pourcentage correspond à la modalité « uniquement des particuliers » donnée en réponse à la question : « Vos clients sont-ils uniquement des particuliers, une seule entreprise, uniquement des entreprises, des entreprises et des particuliers?» 
TABLEAU 1 - Caractéristiques socio-économiques des salarié•e•s et des non-salarié•••s

\begin{tabular}{|c|c|c|c|c|}
\hline & \multicolumn{2}{|c|}{ Non-salariéee·s } & \multicolumn{2}{|c|}{ Salarié·e·s } \\
\hline & Hommes & Femmes & Hommes & Femmes \\
\hline \multicolumn{5}{|l|}{ Secteur d'activité } \\
\hline Agriculture & 18 & 15 & 2 & 1 \\
\hline Industrie & 5 & 4 & 19 & 8 \\
\hline Construction & 18 & 3 & 13 & 2 \\
\hline Tertiaire, dont : & 59 & 78 & 66 & 89 \\
\hline Commerce ; réparation d'automobiles et de motocycles & 15 & 15 & 13 & 12 \\
\hline Hébergement et restauration & 6 & 3 & 3 & 4 \\
\hline $\begin{array}{l}\text { Activités scientifiques et techniques ; services administratifs } \\
\text { et de soutien }\end{array}$ & 14 & 11 & 10 & 9 \\
\hline $\begin{array}{l}\text { Administration publique ; enseignement ; santé humaine } \\
\text { et action sociale }\end{array}$ & 10 & 31 & 21 & 41 \\
\hline Autres activités de services & 4 & 11 & 4 & 11 \\
\hline \multicolumn{5}{|l|}{ Nombre de salarié·e·s employéee·s (pour les non-salarié·e·s) } \\
\hline Aucune & 57 & 72 & & \\
\hline Un.e ou deux & 20 & 15 & & \\
\hline Trois et plus & 23 & 13 & & \\
\hline \multicolumn{5}{|l|}{ Statut juridique } \\
\hline Aide un membre de sa famille & 2 & 6 & & \\
\hline Indépendant·e & 82 & 84 & & \\
\hline Chef.fe d'entreprise & 16 & 10 & & \\
\hline \multicolumn{5}{|l|}{ Niveau de diplôme } \\
\hline Inférieur au bac & 43,5 & 31 & 47 & 37 \\
\hline Bac à bac + 2 & 30,5 & 30 & 29 & 34 \\
\hline Supérieur à bac +2 & 26 & 40 & 24 & 29 \\
\hline
\end{tabular}

Lecture : $18 \%$ des hommes non salariés appartiennent au secteur agricole.

Champ : ensemble des travailleur.euse-s en emploi.

Source : enquête Conditions de travail 2013 (Dares).

ces deux catégories « à activité donnée »n'a de fait qu'un intérêt limité dans la mesure où les deux variables ne sont que partiellement séparables. Seuls quelques secteurs (commerce, services aux particuliers) font cohabiter travailleur.euse-s salarié.e.s et non salarié·e·s mais pour des positions professionnelles elles-mêmes très différentes. Les analyses « toutes choses égales par ailleurs » risqueraient, en ce sens, d'aboutir à des résultats difficilement interprétables.

C'est l'une des raisons qui, compte tenu de la taille de notre échantillon et des objectifs que nous poursuivons (la comparaison des salariées et des non-salariées), nous ont amenés à privilégier le critère de la qualification pour distinguer trois groupes de travailleur·euse·s parmi les non-salariéee·s comme parmi les salarié·e·s : sont ainsi différencié·e·s les actif·ve·s en emploi peu diplômé·e·s (sans diplôme, brevet ou certificat d'aptitude professionnelle [CAP]), celles et ceux détenant un diplôme de niveau intermédiaire (niveau bac à bac +2 ) et enfin celles et ceux ayant obtenu une qualification de niveau bac +3 et au-delà. À l'inverse du secteur d'activité, le niveau 
de diplôme est une dimension transversale, ne dépendant pas du statut juridique de l'emploi. La répartition entre ces trois niveaux de diplôme des non-salariées peut ainsi être comparée à celle qui prévaut chez les salariées : les plus diplômées apparaissent davantage présentes chez les non-salariées ( $40 \%$ contre $29 \%$ ), contrairement aux diplômées de niveau intermédiaire ( $30 \%$ versus $34 \%$ ) et bas ( $31 \%$ versus $37 \%$ ). Cette décomposition de la population des non-salarié.e-s en trois catégories nous permettra de voir que le statut juridique de l'activité a des effets comparables selon le niveau de formation en matière de temps de travail.

Avant cela, précisons les principales caractéristiques des sous-populations ainsi identifiées.

\section{Caractéristiques socio-démographiques et professionnelles des non-salariées en fonction de leur niveau de diplôme}

En dépit de l'hétérogénéité de la catégorie des non-salarié·e·s, deux grandes tendances demeurent marquantes : la population des non-salariée-s s est sensiblement plus âgée que celle des salarié.e.s (tant en raison de leurs trajectoires individuelles que des secteurs auxquels elles et ils appartiennent) et les femmes y sont fortement sous-représentées (seules $30 \%$ des non-salarié.e-s sont des femmes). Cette proportion est équivalente à celle observée dans l'édition 2005 de l'enquête Conditions de travail. Au-delà de ces caractéristiques communes, comment les sous-populations que définissent le statut juridique et le niveau de diplôme se distinguent-elles et, plus spécifiquement, comment, au sein de chacune d'elles, les femmes salariées et non salariées se différencient-elles ? Nous nous arrêtons ici sur deux éléments fortement liés à l'organisation des temps de travail : la situation familiale et la «famille professionnelle ${ }^{5} \gg$ (tableau 2).

Dans la sous-population des peu ou pas diplômées (diplôme inférieur au bac), le statut de non-salarié est relativement peu fréquent : moins de $7 \%$ d'entre elles exercent une activité professionnelle non salariée et parmi elles, la grande majorité (79 \%) sont des travailleuses indépendantes et une sur dix « aide un membre de sa famille sans être rémunérée », principalement dans l'agriculture ou le commerce (estimations non reproduites dans le tableau 2). La moyenne d'âge des non-salariées est par ailleurs sensiblement plus élevée pour les faiblement diplômées, la part des 50 ans et plus y dépassant $55 \%$, contre respectivement $30 \%$ et $35,5 \%$ pour les catégories plus diplômées et moins de $45 \%$ chez les salariées ayant un niveau de diplôme équivalent. Ces différences d'âge se répercutent sur les configurations familiales : plus souvent en couple sans enfant, elles sont au contraire sensiblement moins fréquemment

\footnotetext{
5. «Les familles professionnelles » regroupent les professions qui font appel à des compétences communes sur la base de "gestes professionnels" proches » (cf. Dares, La Nomenclature des familles professionnelles. Version 2009. Table de correspondance FAP/PCS/ROME, en ligne : http://travail-emploi.gouv.fr/IMG/pdf/FAP-2009_Introduction_ et_table_de_correspondance.pdf, consulté le 13 octobre 2017). Elles permettent d'analyser les données du marché du travail provenant de différentes sources (Insee, Pôle Emploi, etc.).
} 


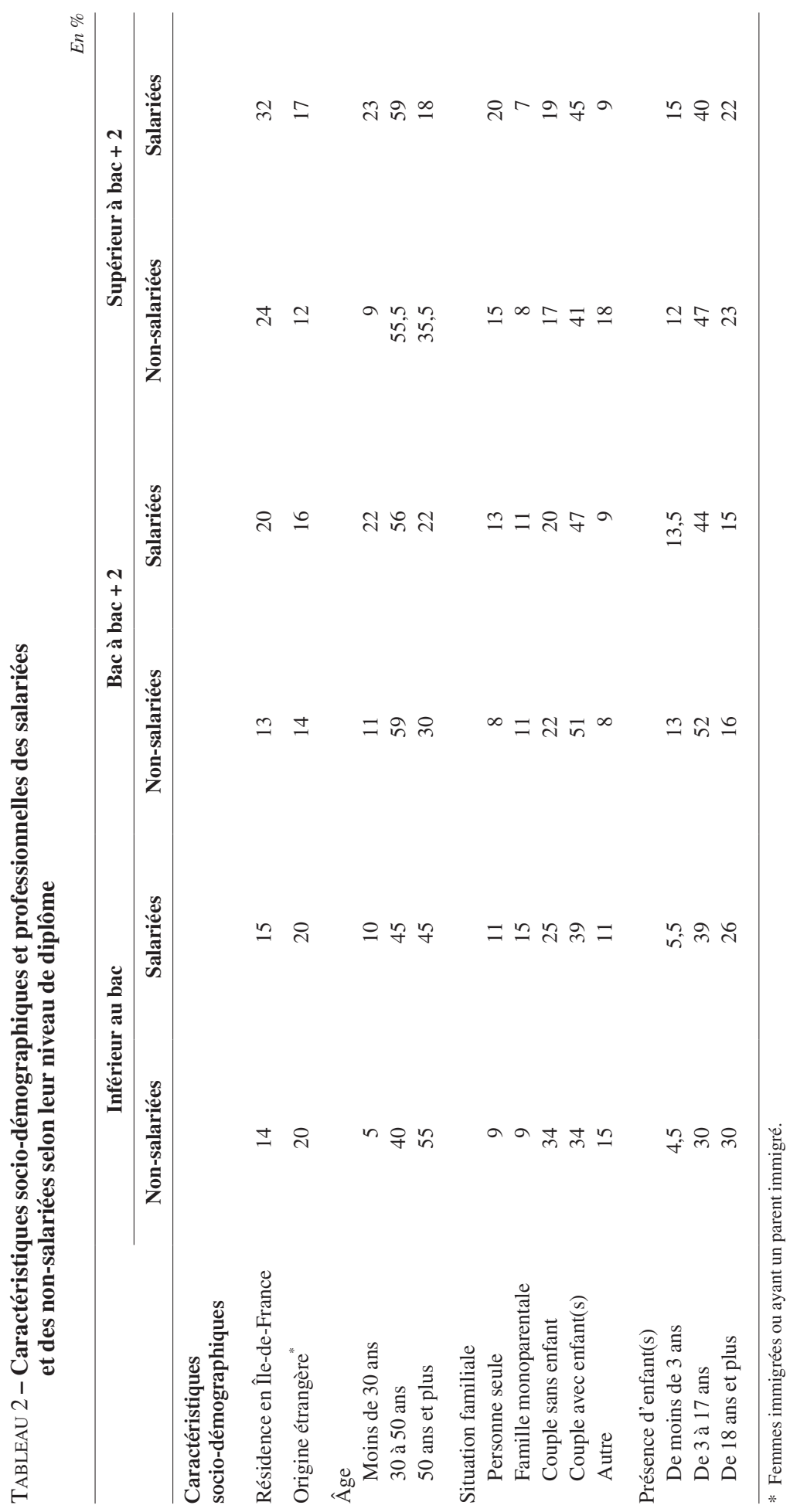


Femmes salariées et non salariées : quelles différences de temps de travail ?

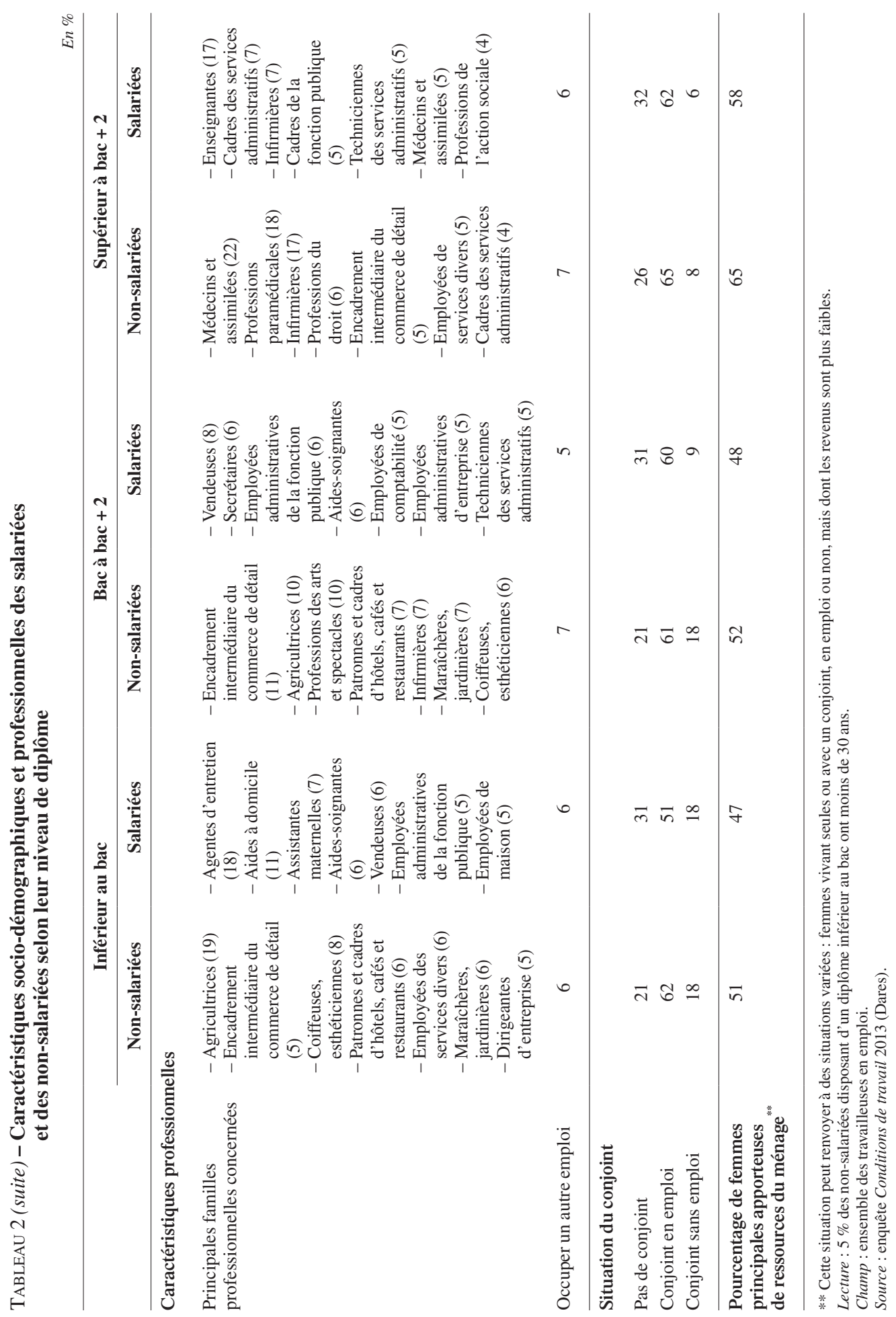


responsables d'une famille monoparentale ou en couple avec enfants. Lorsqu' un enfant est présent, il est par ailleurs plus âgé. Ces non-salariées peu diplômées se retrouvent principalement dans un nombre réduit de familles professionnelles : l'agriculture occupe une place conséquente (environ un quart de ces non-salariées) tout comme le commerce (principalement des commerçantes, un cinquième des non-salariées) et les services aux particuliers (notamment en tant que coiffeuses, esthéticiennes, patronnes de bar ou de restaurant, etc.) pour un cinquième des non-salariées à nouveau. Du côté des salariées, ce sont également les métiers des services aux particuliers qui sont fortement représentés (assistantes maternelles, aides à domicile, caissières, coiffeuses salariées, vendeuses, etc.), mais une part également élevée relève des services publics.

Les constats effectués sur les travailleuses peu diplômées sont également en partie vrais pour celles détenant un diplôme de niveau bac à bac + 2. Dans ce groupe, les non-salariées sont de même plus âgées et plus souvent en couple que leurs homologues salariées. Elles ont par contre plus souvent des enfants mineurs à charge (65\%, contre $57 \%$ ). Sans disparaître, le poids des métiers agricoles se réduit sensiblement (moins d'une non-salariée sur six) tandis que les emplois du commerce et des services aux particuliers demeurent centraux : ils concernent chacun environ un quart des nonsalariées de qualification intermédiaire. Les professions des arts et des spectacles, mais aussi médicales et paramédicales occupent par ailleurs également une proportion importante de ces femmes (environ $10 \%$ chacune).

Chez les travailleuses disposant d'un diplôme supérieur à bac +2 , la surreprésentation des 50 ans et plus est encore plus forte chez les non-salariées que chez les salariées. Les écarts observables en matière de configurations familiales sont en revanche assez comparables aux travailleuses moins diplômées : les non-salariées sont légèrement moins souvent seules et ont des enfants plus âgés. Si pour toutes les catégories de qualification, les non-salariées sont un peu plus souvent la principale apporteuse de ressources du ménage (ce qui peut néanmoins renvoyer à des situations variées : femmes vivant seules ou avec un conjoint, en emploi ou non, mais dont les revenus sont plus faibles), cette tendance se renforce nettement chez les plus diplômées : 65 \% des non-salariées occupent cette position, contre 58 \% des salariées. En termes de secteurs de familles professionnelles, la spécialisation dans le domaine médical et paramédical apparaît nettement (ces emplois regroupent plus de $56 \%$ de ces non-salariées), devant les autres professions libérales (notamment juridiques) et les métiers commerciaux.

Bien que les trois groupes de non-salariées que nous avons isolés en fonction du niveau de diplôme renvoient à des configurations diverses, notamment en matière de familles professionnelles, leurs temps de travail partagent de nombreux points communs. 


\section{Une disponibilité temporelle au travail particulièrement élevée}

L'analyse du temps de travail des non-salariées à partir de l'enquête Conditions de travail fait apparaître plusieurs faits marquants : des durées plus longues, des horaires atypiques plus fréquents, des emplois du temps moins prévisibles et plus poreux visà-vis de la vie personnelle.

\section{Des durées plus longues}

Les écarts entre les femmes non salariées et salariées sont très proches de ceux observés entre leurs homologues masculins : pour les unes comme pour les autres être à son compte implique une durée de travail hebdomadaire bien plus longue. En 2013, les femmes non salariées travaillent en moyenne $41 \mathrm{~h} 20$ par semaine (contre $33 \mathrm{~h} 45$ pour les salariées). Ces durées sont ainsi nettement supérieures à celles des salariées, y compris celles des plus diplômées dont la durée hebdomadaire moyenne dépasse légèrement 37 heures (tableau 3).

Les durées moyennes de travail des non-salariées sont nettement plus élevées que celles des salariées mais elles apparaissent également sensiblement plus dispersées. Les « longues durées » sont ainsi plus fréquentes ( $25 \%$ des non-salariées travaillent plus de 50 heures par semaine), mais elles coexistent avec des durées plus courtes,

TABLEAU 3 - Durée de travail hebdomadaire des salarié·e·s et des non-salarié·e·s selon leur niveau de diplôme

\begin{tabular}{|c|c|c|c|c|c|c|}
\hline & Moyenne & $\begin{array}{l}\text { Premier } \\
\text { quartile }\end{array}$ & Médiane & $\begin{array}{l}\text { Troisième } \\
\text { quartile }\end{array}$ & $\begin{array}{c}\text { Rapport } \\
\text { interquartile }\end{array}$ & $\begin{array}{c}\text { Nombre } \\
\text { d'obs. }\end{array}$ \\
\hline \multicolumn{7}{|l|}{ Femmes non salariées } \\
\hline Inférieur au bac & 41 h 31 & $30 \mathrm{~h}$ & $40 \mathrm{~h}$ & $55 \mathrm{~h}$ & 1,83 & 415 \\
\hline Bac à bac + 2 & 37 h 56 & $25 \mathrm{~h}$ & $40 \mathrm{~h}$ & $50 \mathrm{~h}$ & 2,00 & 322 \\
\hline Supérieur à bac + 2 & 43 h 39 & $35 \mathrm{~h}$ & $44 \mathrm{~h}$ & $50 \mathrm{~h}$ & 1,43 & 346 \\
\hline \multicolumn{7}{|l|}{ Femmes salariées } \\
\hline Inférieur au bac & $31 \mathrm{~h} 00$ & $24 \mathrm{~h}$ & $35 \mathrm{~h}$ & $37 \mathrm{~h}$ & 1,54 & 5816 \\
\hline Bac à bac + 2 & 33 h 48 & $30 \mathrm{~h}$ & $35 \mathrm{~h}$ & $39 \mathrm{~h}$ & 1,30 & 5177 \\
\hline Supérieur à bac + 2 & 37 h 03 & $34 \mathrm{~h}$ & $38 \mathrm{~h}$ & $42 \mathrm{~h}$ & 1,24 & 4136 \\
\hline \multicolumn{7}{|l|}{ Hommes non salariés } \\
\hline Inférieur au bac & 50 h 46 & $40 \mathrm{~h}$ & $50 \mathrm{~h}$ & $65 \mathrm{~h}$ & 1,63 & 1033 \\
\hline Bac à bac + 2 & 52 h 20 & $42 \mathrm{~h}$ & $50 \mathrm{~h}$ & $65 \mathrm{~h}$ & 1,55 & 581 \\
\hline Supérieur à bac + 2 & 48 h 20 & $40 \mathrm{~h}$ & $50 \mathrm{~h}$ & $56 \mathrm{~h}$ & 1,40 & 427 \\
\hline \multicolumn{7}{|l|}{ Hommes salariés } \\
\hline Inférieur au bac & 38 h 00 & $35 \mathrm{~h}$ & $37 \mathrm{~h}$ & $40 \mathrm{~h}$ & 1,14 & 5571 \\
\hline Bac à bac + 2 & 39 h 05 & $35 \mathrm{~h}$ & $39 \mathrm{~h}$ & $42 \mathrm{~h}$ & 1,20 & 3355 \\
\hline Supérieur à bac + 2 & 42 h 23 & $38 \mathrm{~h}$ & $40 \mathrm{~h}$ & $50 \mathrm{~h}$ & 1,32 & 2697 \\
\hline
\end{tabular}

Lecture : la durée hebdomadaire moyenne de travail des femmes non salariées ayant un niveau de diplôme inférieur au bac est 41 h 31 , soit 10 h 31 de plus que la durée hebdomadaire moyenne des femmes salariées de même niveau de diplôme.

Champ : ensemble des travailleur.euse-s en emploi.

Source : enquête Conditions de travail 2013 (Dares). 
équivalentes à des emplois à temps partiel, également déclarées dans un quart des cas. Les inégalités de temps de travail, mesurées ici par les rapports interquartiles, sont également sensiblement plus élevées chez les non-salariées. Cette variété des situations, qui touchent toutes les catégories de diplôme et les femmes comme les hommes, peuvent renvoyer à une dépendance accrue vis-à-vis de contraintes marchandes, mais également à l'existence de marges de manœuvre plus grandes pour faire varier les durées de travail.

Un niveau de qualification croissant est associé à des durées plus longues chez les salariées alors que l'effet est plus ambigu pour les non-salariées : les durées longues s'observent ainsi à la fois chez les moins diplômées et chez celles qui le sont le plus ; et ce sont les femmes ayant un niveau intermédiaire de qualification pour lequel l'écart de durée est le plus faible entre salariées et non-salariées. Ces résultats s'expliquent par les familles professionnelles des emplois concernés : pour les moins diplômées, les emplois agricoles sont associés à des durées particulièrement longues tout comme les activités médicales pour les plus diplômées.

\section{Des journées de travail plus étendues}

Ces durées hebdomadaires se répercutent logiquement sur l'étendue des journées de travail. Ainsi, en nous basant sur les questions : «Habituellement, à quelle heure commencez-vous votre travail ? » et : « Habituellement, à quelle heure terminez-vous votre travail ? », nous avons calculé la proportion de travailleuses « au travail » sur chaque quart d'heure de la journée. Sur cette base, nous dressons la courbe de présence au travail sur vingt-quatre heures. Elle permet de repérer les créneaux horaires dédiés au travail professionnel (graphique 1).

La journée de travail des non-salariées apparaît bien comme plus étendue que celle des salariées. Les écarts de taux de présence sont visibles en début de journée (de $6 \mathrm{~h} 30$ à 8 heures), mais ils sont surtout particulièrement élevés en fin de journée : alors que deux non-salariées sur trois sont au travail à $18 \mathrm{~h} \mathrm{00,} \mathrm{ce} \mathrm{n'est} \mathrm{le} \mathrm{cas} \mathrm{que} \mathrm{de} \mathrm{moins} \mathrm{d'un} \mathrm{tiers}$ des salariées. Ces écarts sont particulièrement importants entre non-salariées et salariées diplômées (graphique 4), ce qui est en grande partie dû aux professions médicales ${ }^{6}$.

Plus largement, les graphiques 2, 3 et 4 font bien ressortir les configurations journalières spécifiques de chaque sous-population de femmes, salariées et non salariées, et selon leur niveau de diplôme. Par rapport à la norme statistique que représente la journée des salariées les plus diplômées - c'est la plus proche d'une journée « standard » de travail s'étalant de $8 \mathrm{~h}$ à $18 \mathrm{~h}$-, trois périodes peuvent être distinguées : le petit matin, où les femmes non qualifiées (salariées ou non) sont davantage présentes ; le cœur de la journée, où toutes les catégories de travailleuses sont moins présentes que les salariées les plus diplômées, avec un écart particulièrement important pour les non-qualifiées, et encore davantage pour celles qui sont salariées ; la fin d'après-midi et la soirée, enfin, où les non-salariées sont surreprésentées, quel que soit leur niveau de qualification.

6. Un phénomène comparable est observé chez les hommes. 
GRAPHIQUE 1 - La présence au travail, heure par heure, des salariées et des non-salariées

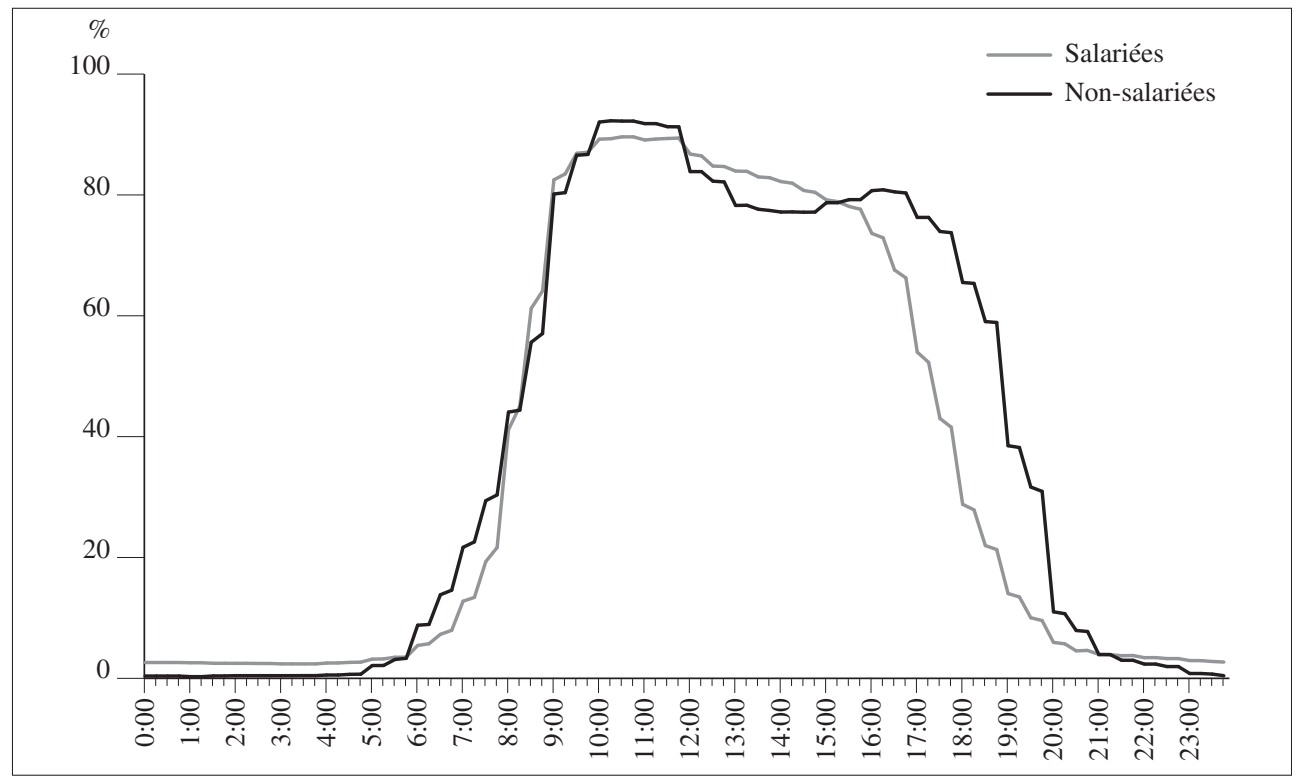

Lecture : en 2013,44,1\% des femmes non salariées et 41,2\% des femmes salariées déclaraient être habituellement au travail à 8 h 00 . Champ : ensemble des travailleuses en emploi.

Source : enquête Conditions de travail 2013 (Dares).

GRAPHIQUE 2 - La présence au travail, heure par heure, des salariées et des non-salariées, dont le niveau de diplôme est inférieur au bac

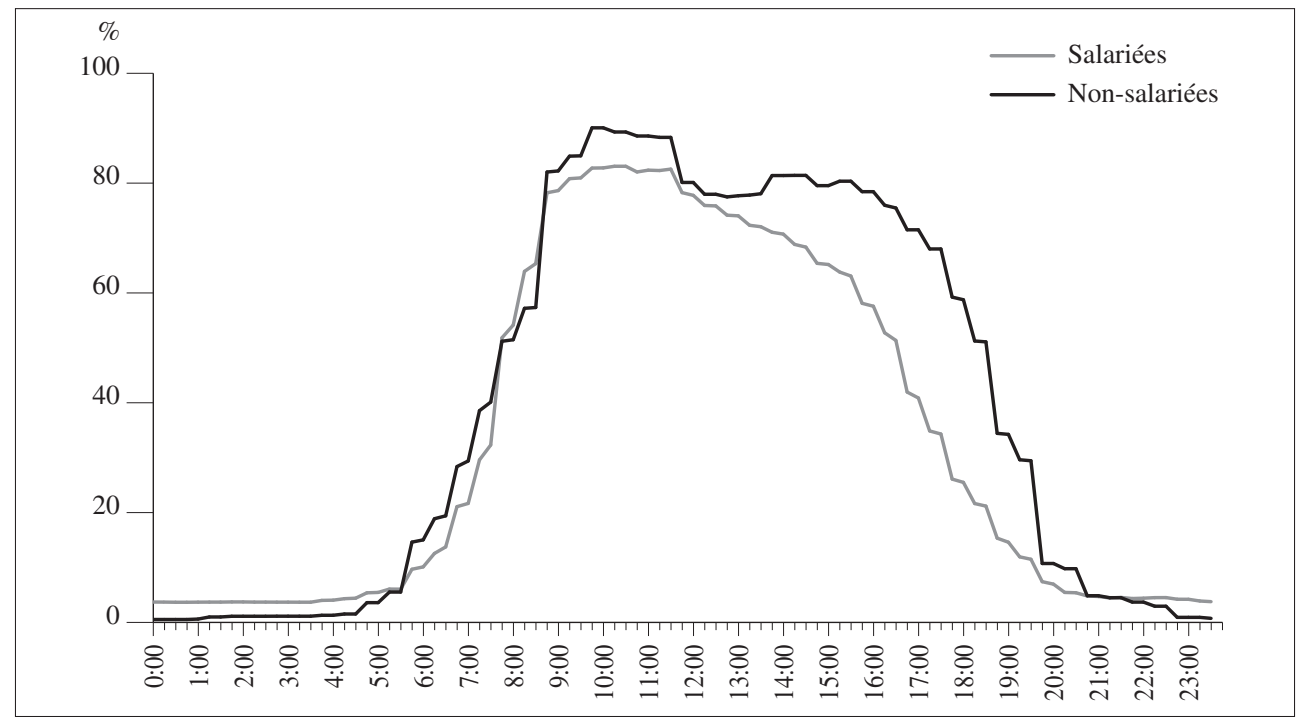

Lecture : en 2013, 51,5\% des femmes non salariées ayant un niveau de diplôme inférieur au bac déclaraient être habituellement au travail à $8 \mathrm{~h} 00$, contre $54,2 \%$ des femmes salariées de niveau de diplôme équivalent.

Champ : ensemble des travailleuses ayant un niveau de diplôme inférieur au bac en emploi.

Source : enquête Conditions de travail 2013 (Dares). 
GRAPHIQUE 3 - La présence au travail, heure par heure, des salariées et des non-salariées, dont le niveau de diplôme est compris entre bac et bac +2

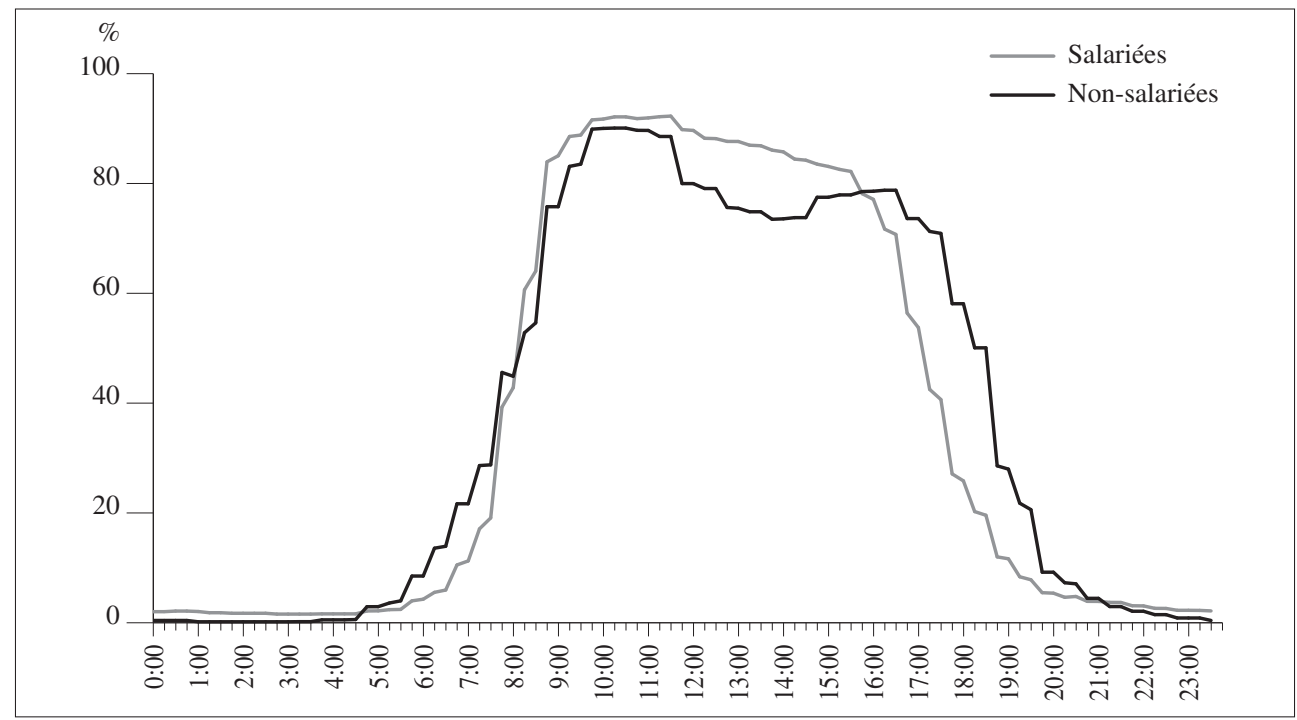

Lecture : en 2013, 44,9 \% des femmes non salariées ayant un niveau de diplôme compris entre bac et bac + 2 déclaraient être habituellement au travail à $8 \mathrm{~h} 00$, contre $42,8 \%$ des femmes salariées de niveau de diplôme équivalent.

Champ : ensemble des travailleuses en emploi ayant un niveau de diplôme compris entre bac et bac +2 .

Source : enquête Conditions de travail 2013 (Dares).

GRAPHIQUE 4 - La présence au travail, heure par heure, des salariées et des non-salariées, dont le niveau de diplôme est supérieur à bac +2

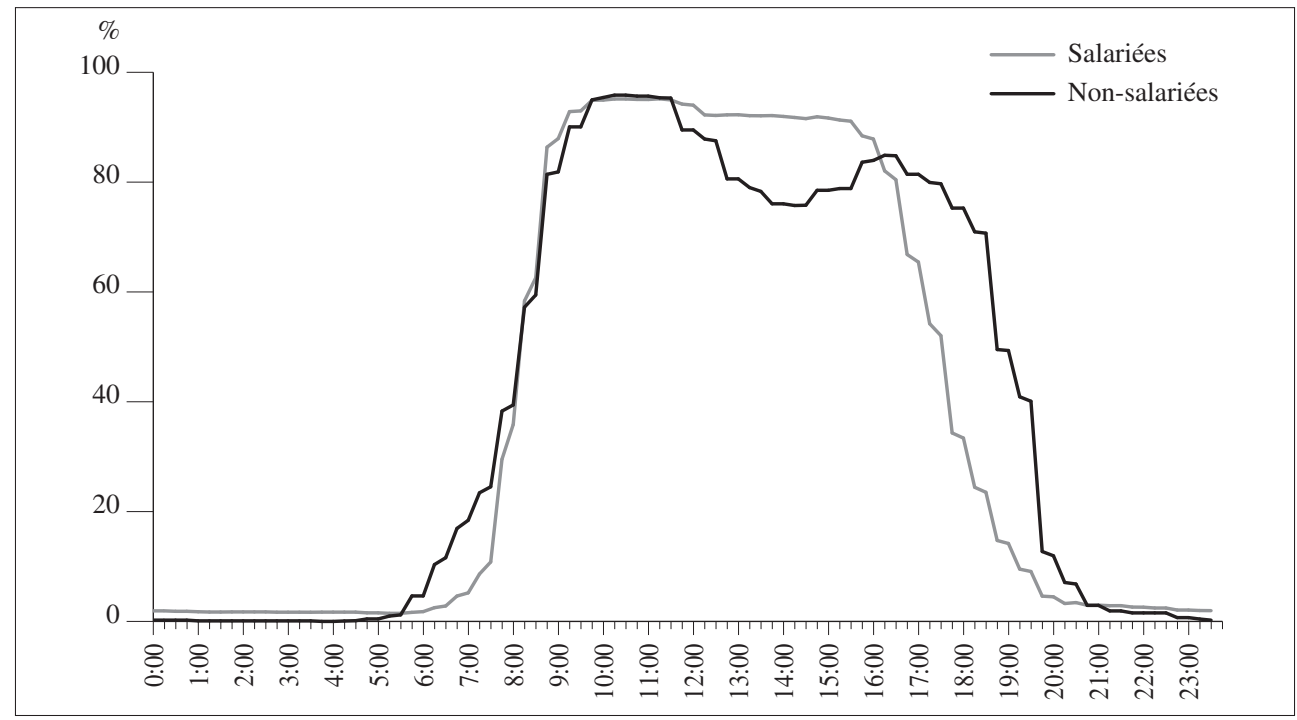

Lecture : en 2013, 39,4 \% des femmes non salariées ayant un niveau de diplôme supérieur à bac +2 déclaraient être habituellement au travail à $8 \mathrm{~h} 00$, contre $35,9 \%$ des femmes salariées de niveau de diplôme équivalent.

Champ : ensemble des travailleuses en emploi ayant un niveau de diplôme supérieur à bac +2 .

Source : enquête Conditions de travail 2013 (Dares). 
GRAPHIQUE 5 - Écarts de présence au travail, heure par heure, entre non-salariées et salariées, selon le niveau de diplôme

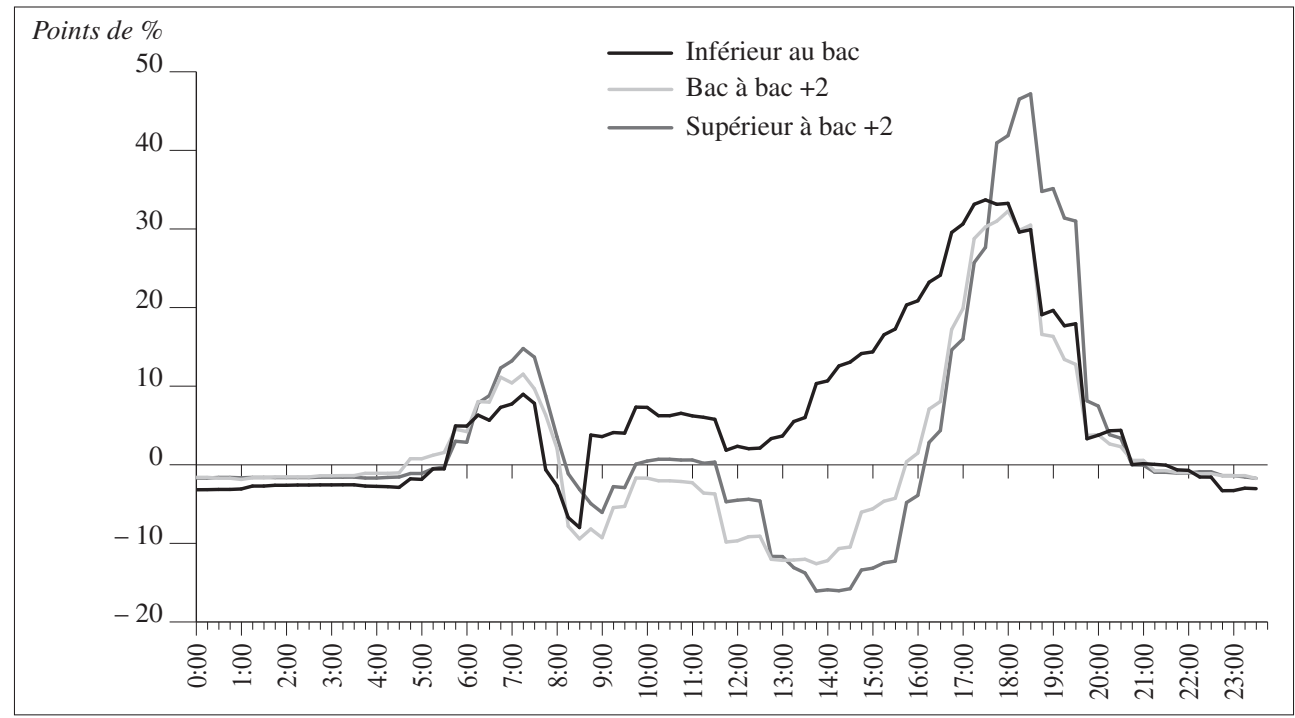

Lecture : en 2013, à 18 h 00, le taux de présence des femmes non salariées ayant un niveau de diplôme inférieur au bac est supérieur de 30 points au taux de présence des femmes salariées de niveau de diplôme équivalent.

Champ : ensemble des travailleuses en emploi.

Source : enquête Conditions de travail 2013 (Dares).

À niveau de diplôme donné (graphique 5), les écarts entre non-salariées et salariées sont proches pour les niveaux de diplôme intermédiaires et élevés : on y observe une présence moins forte des femmes à leur compte en milieu de journée et, à l'inverse, une présence plus marquée en début et, surtout, en fin de journée. Une part importante de l'activité des non-salariées a donc lieu de façon décalée avec les horaires standards de travail. Il s'agit pour partie des activités de services qui s'adressent aux particuliers (commerce, santé libérale).

Chez les non-diplômées, la situation est différente : la présence des non-salariées est plus importante pratiquement tout au long de la journée (à l'exception d'une heure, entre 8 heures et 9 heures le matin), et tout particulièrement à partir du milieu de l'aprèsmidi (plus de 20 points de taux de présence dès 14 h 30 et jusqu'à 19 h 45). On peut faire l'hypothèse que pour les femmes non diplômées, le travail est décompté de manière sensiblement différente entre salariées et non-salariées. L'activité des secondes apparaît davantage extensive - elle occupe l'ensemble de la journée, y compris des périodes plus « creuses »-, tandis que pour les salariées, ces périodes « creuses » sortent du temps travaillé et ont pour conséquence des emplois du temps plus fractionnés et des durées de travail effectif plus courtes. Comme nous le verrons, ces deux mécanismes de gestion d'une activité morcelée ont des répercussions assez proches en termes de rémunérations. 
L'analyse des journées de travail permet de calculer l'étendue de la journée de travail (c'est-à-dire, la différence entre l'horaire de fin et l'horaire de début du travail) et la « densité » de cette journée, à savoir le ratio entre la durée quotidienne moyenne (durée hebdomadaire divisée par le nombre de jours de travail hebdomadaire) et l'étendue de la journée (tableau 4). Cet indicateur de densité révèle des différences sensibles : une densité globalement plus faible pour les non-salarié.e.s (femmes et hommes) par rapport aux salarié.e.s qui témoigne d'un « creux » plus important au milieu de la journée. Celui-ci correspond le plus souvent à une pause méridienne plus longue mais avec différentes facettes possibles (repos pour ceux qui commencent très tôt ou déjeuner d'affaire par exemple). Des densités aussi faibles ne se retrouvent par ailleurs que dans certaines professions salariées peu qualifiées telles que les agent.e.s du nettoyage, les aides à domiciles ou dans le secteur de l'hôtellerie-restauration. Les femmes non salariées les plus diplômées constituent une exception notable : elles connaissent des étendues journalières plus faibles que les hommes non salariés pour des durées en revanche quasi identiques. Cette situation peut renvoyer aux obligations familiales, notamment parentales, qui continuent de toujours peser majoritairement sur les femmes.

TABLEAU 4 - Durée quotidienne moyenne, étendue et densité des journées de travail des salariée's et des non-salarié·e·s selon leur niveau de diplôme

\begin{tabular}{|c|c|c|c|}
\hline & Étendue journalière & $\begin{array}{c}\text { Durée quotidienne } \\
\text { moyenne }\end{array}$ & $\begin{array}{c}\text { Densité de la journée } \\
\text { de travail }(\%)\end{array}$ \\
\hline \multicolumn{4}{|l|}{ Femmes non salariées } \\
\hline Inférieur au bac & $10 \mathrm{~h} 06$ & $7 \mathrm{~h} 30$ & 74 \\
\hline Bac à bac + 2 & $9 \mathrm{~h} 48$ & 7 h 18 & 75 \\
\hline Supérieur à bac + 2 & $10 \mathrm{~h} 30$ & 9 h 18 & 89 \\
\hline Ensemble & $10 \mathrm{~h} 12$ & $8 \mathrm{~h} 12$ & 80 \\
\hline \multicolumn{4}{|l|}{ Femmes salariées } \\
\hline Inférieur au bac & $8 \mathrm{~h} 18$ & $6 \mathrm{~h} 42$ & 80 \\
\hline Bac à bac + 2 & 8 h 54 & 7 h 24 & 83 \\
\hline Supérieur à bac + 2 & 9 h 12 & $8 \mathrm{~h} 00$ & 87 \\
\hline Ensemble & 8 h 48 & $7 \mathrm{~h} 18$ & 83 \\
\hline \multicolumn{4}{|l|}{ Hommes non salariés } \\
\hline Inférieur au bac & $10 \mathrm{~h} 48$ & 8 h 54 & 82 \\
\hline Bac à bac + 2 & $11 \mathrm{~h} 00$ & 9 h 18 & 85 \\
\hline Supérieur à bac + 2 & $11 \mathrm{~h} 00$ & 9 h 18 & 85 \\
\hline Ensemble & 10 h 54 & $9 \mathrm{~h} 06$ & 84 \\
\hline \multicolumn{4}{|l|}{ Hommes salariés } \\
\hline Inférieur au bac & 9 h 12 & 7 h 42 & 84 \\
\hline Bac à bac + 2 & 9 h 24 & $8 \mathrm{~h} 00$ & 85 \\
\hline Supérieur à bac + 2 & 9 h 42 & 8 h 36 & 88 \\
\hline Ensemble & 9 h 24 & $8 \mathrm{~h} 00$ & 85 \\
\hline
\end{tabular}

Note : la densité de la journée de travail correspond au ratio entre la durée quotidienne et l'étendue journalière. Elle permet d'évaluer le fractionnement (ou le morcellement) du temps de travail, c'est-à-dire le rapport entre le temps effectivement travaillé (et donc rémunéré) et l'amplitude globale de la journée de travail.

Lecture : les femmes non salariées ont des amplitudes quotidiennes de travail de 10 heures 12 minutes pour des durées quotidiennes moyennes de 8 heures 12 minutes. Cette durée quotidienne représente $80 \%$ de leur amplitude journalière.

Champ : ensemble des travailleur.euse-s en emploi.

Source : enquête Conditions de travail 2013 (Dares). 


\section{Des temps plus atypiques}

Les non-salariées sont nettement plus concernées par les horaires décalés, en début et en fin de journée, nous l'avons vu, mais aussi en dehors des heures habituelles de travail (tableau 5) : elles sont ainsi plus nombreuses à travailler (occasionnellement ou régulièrement) le soir (42\%, contre $25 \%$ des salariées), la nuit ( $11 \%$, contre $8 \%{ }^{7}$ ), ainsi que le samedi $(81 \%$, contre $42 \%)$ et le dimanche (54\%, contre $24 \%)$.

\section{TABLEAU 5 - Horaires atypiques (habituels ou occasionnels) des salarié·e•s et des non-salarié•e·s selon leur niveau de diplôme}

\begin{tabular}{|c|c|c|c|c|c|}
\hline & $\begin{array}{c}\text { Matin } \\
(5 \mathrm{~h} 00-7 \mathrm{~h} 00)\end{array}$ & $\begin{array}{c}\text { Soir } \\
(20 \text { h } 00 \text {-minuit })\end{array}$ & $\begin{array}{c}\text { Nuit } \\
\text { (minuit-5 h 00) }\end{array}$ & Samedi & Dimanche \\
\hline \multicolumn{6}{|l|}{ Femmes non salariées } \\
\hline Inférieur au bac & 30,6 & 31,4 & 10,5 & 83,1 & 54,4 \\
\hline Bac à bac + 2 & 23,4 & 37,1 & 9,1 & 79,2 & 49,7 \\
\hline Supérieur à bac + 2 & 27,1 & 54,6 & 13,9 & 80,3 & 58,7 \\
\hline Ensemble & 27,0 & 42,0 & 11,0 & 81,0 & 54,0 \\
\hline \multicolumn{6}{|l|}{ Femmes salariées } \\
\hline Inférieur au bac & 20,2 & 19,3 & 7,4 & 45,6 & 25,6 \\
\hline Bac à bac + 2 & 13,2 & 20,7 & 7,3 & 40,9 & 23,2 \\
\hline Supérieur à bac + 2 & 13,0 & 36,7 & 8,9 & 39,3 & 23,4 \\
\hline Ensemble & 16,0 & 25,0 & 8,0 & 42,0 & 24,0 \\
\hline \multicolumn{6}{|l|}{ Hommes non salariés } \\
\hline Inférieur au bac & 43,0 & 44,0 & 24,2 & 86,0 & 50,0 \\
\hline Bac à bac + 2 & 49,6 & 62,2 & 28,8 & 85,6 & 64,2 \\
\hline Supérieur à bac + 2 & 33,0 & 61,5 & 22,8 & 78,8 & 54,4 \\
\hline Ensemble & 42,0 & 54,0 & 25,0 & 84,0 & 55,0 \\
\hline \multicolumn{6}{|l|}{ Hommes salariés } \\
\hline Inférieur au bac & 37,8 & 29,4 & 20,4 & 47,9 & 25,3 \\
\hline $\mathrm{Bac}$ à bac + 2 & 32,6 & 36,0 & 19,4 & 51,8 & 31,2 \\
\hline Supérieur à bac + 2 & 20,6 & 43,4 & 13,5 & 43,4 & 28,9 \\
\hline Ensemble & 32,0 & 35,0 & 28,0 & 48,0 & 28,0 \\
\hline
\end{tabular}

Note : l'enquête Conditions de travail 2013 nous permet de distinguer cinq périodes de travail atypique : le matin (entre $5 \mathrm{~h} 00$ et $7 \mathrm{~h} 00$ );

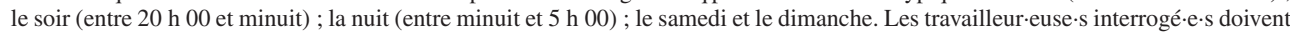
répondre à la question suivante : "Travaillez-vous la nuit, entre minuit et cinq heures du matin ? », en choisissant eux·elles-mêmes la fréquence : «Habituellement/Occasionnellement/Jamais ». La formulation de la question est identique pour les cinq périodes. Les modalités de réponse ne correspondent pas à des paliers : les enquêté-e·s doivent se classer eux·elles-mêmes. L'appréciation de ce qui est une pratique habituelle (ou occasionnelle) peut changer d'un·e travailleur-euse à l'autre.

Nous considérons ici que les travailleur·euse-s travaillent en horaires atypiques lorsqu'ils·elles ont déclaré travailler « habituellement » ou « occasionnellement » sur ces plages horaires.

Lecture : en 2013, 30,6 \% des femmes non salariées ayant un niveau de diplôme inférieur au bac travaillent habituellement ou occasionnellement le matin, entre $5 \mathrm{~h} 00$ et $7 \mathrm{~h} 00$.

Champ : ensemble des travailleur-euse-s en emploi.

Source : enquête Conditions de travail 2013 (Dares).

7. Les effets sont cependant plus nuancés lorsque l'on distingue le travail occasionnel et le travail régulier durant ces périodes atypiques : le travail de nuit régulier est ainsi plus fréquent chez les salariées. 
En la matière, les différences observées entre les sexes sont les mêmes pour les salarié.e.s et les non-salarié.e.s : les hommes sont davantage concernés par le travail du soir et de la nuit, tandis que les femmes le sont par les autres horaires atypiques (le matin et le week-end). Enfin, le niveau de qualification semble également intervenir de manière symétrique selon que l'activité est exercée à son compte ou non : à l'exception du travail le soir, un diplôme plus élevé limite les horaires s'écartant des rythmes collectifs et habituels de travail.

Certaines catégories sont ainsi très fortement touchées par des périodes de travail atypiques : c'est notamment le cas des femmes non salariées et non diplômées, qui travaillent à près de $84 \%$ le samedi, contre moins de $46 \%$ des femmes salariées de niveau de qualification équivalent. À nouveau, le poids des activités agricoles et commerciales est ici directement repérable.

Au final, ces éléments (horaires atypiques, étendues des journées et longues durées) tendent à se cumuler plus encore chez les non-salariées que chez les salariées. Chez ces dernières, une forme de substitution entre durée et horaires fragmentés ou atypiques peut parfois apparaitre (DEVETTER, 2002) : en effet, alors que les salariées travaillant habituellement le dimanche ont une durée hebdomadaire moyenne quasiment identique à celle des autres salariées, chez les non-salariées, le travail dominical est associé à des durées bien supérieures (43 heures, contre 33 heures). Ce constat se retrouve pour l'ensemble des horaires atypiques, qui constituent ainsi des « heures en plus » pour les non-salarié.e.s (femmes comme hommes d'ailleurs) alors qu'ils se rapprochent « d'heures à la place » d'autres heures pour les salariéee·s.

\section{Des horaires moins prévisibles mais mieux maîtrisés}

Longs et décalés, les temps de travail des non-salariées sont également bien plus imprévisibles que ceux des salariées : alors que $84 \%$ de ces dernières connaissent leurs horaires du mois à venir, ce n'est le cas que de $60 \%$ des non-salariées. Pourtant, l'une des caractéristiques majeures de leur temps de travail est la très grande latitude dont elles disposent a priori dans l'organisation de leur travail et de leurs horaires. En effet, elles sont plus de $90 \%$ à déclarer qu'elles ont déterminé elles-mêmes leurs horaires (contre $12 \%$ des salariées). En outre, du fait même de la nature de leur activité et de leur statut, elles ne sont soumises à aucun contrôle horaire (c'est le cas de $95 \%$ d'entre elles, contre $56 \%$ des salariées). Cette autonomie se traduit par une plus grande capacité à organiser son travail selon les convenances personnelles (tableau 6).

La possibilité de gérer des imprévus par des absences n'est pas considérée comme plus facile pour les non-salariées prises dans leur ensemble. Ce constat est le résultat de deux situations opposées : alors que chez les plus diplômées, les non-salariées sont particulièrement contraintes, chez les non-diplômées, le statut de non-salariée permet une souplesse bien plus importante. La possibilité de « prendre sur son temps de travail » semble être une ressource non négligeable, qui vient compenser de multiples contraintes (durée, étendue et imprévisibilité des horaires) qui pèsent sur le temps de travail de ces non-salariées. 
TABLEAU 6 - Prévisibilité et maîtrise des horaires des salarié·e•s et des non-salarié•e•s selon leur niveau de diplôme

\begin{tabular}{|c|c|c|c|}
\hline & $\begin{array}{c}\text { «Connaissez-vous } \\
\text { les horaires que vous } \\
\text { devrez effectuer dans } \\
\text { le mois à venir ? " } \\
(\text { (« oui ») }\end{array}$ & $\begin{array}{c}\text { «Je peux organiser } \\
\text { mon travail de la manière } \\
\text { qui convient. » } \\
\text { («tout à fait d'accord ») }\end{array}$ & $\begin{array}{l}\text { « En cas d'imprévu } \\
\text { personnel ou familial, } \\
\text { pouvez-vous vous absenter } \\
\text { de votre travail, même } \\
\text { quelques heures? } \\
\text { («oui, c'est facile » ou } \\
\text { « oui, mais ce n'est pas } \\
\text { facile») }\end{array}$ \\
\hline \multicolumn{4}{|l|}{ Femmes non salariées } \\
\hline Inférieur au bac & 57 & 57 & 72 \\
\hline Bac à bac + 2 & 60 & 46 & 68 \\
\hline Supérieur à bac + 2 & 63 & 58 & 52 \\
\hline Ensemble & 60 & 54 & 63 \\
\hline \multicolumn{4}{|l|}{ Femmes salariées } \\
\hline Inférieur au bac & 82 & 29 & 60 \\
\hline Bac à bac + 2 & 85 & 30 & 61 \\
\hline Supérieur à bac + 2 & 86 & 31 & 61 \\
\hline Ensemble & 84 & 29 & 61 \\
\hline \multicolumn{4}{|l|}{ Hommes non salariés } \\
\hline Inférieur au bac & 50 & 46 & 75 \\
\hline Bac à bac + 2 & 50 & 48 & 77 \\
\hline Supérieur à bac + 2 & 53 & 53 & 69 \\
\hline Ensemble & 51 & 48 & 74 \\
\hline \multicolumn{4}{|l|}{ Hommes salariés } \\
\hline Inférieur au bac & 76 & 27 & 66 \\
\hline Bac à bac + 2 & 76 & 34 & 72 \\
\hline Supérieur à bac + 2 & 77 & 39 & 75 \\
\hline Ensemble & 76 & 31 & 70 \\
\hline
\end{tabular}

Lecture : en 2013, 57 \% des femmes non salariées ayant un niveau de diplôme inférieur au bac connaissent leurs horaires au moins un mois à l'avance.

Champ : ensemble des travailleur·euse-s en emploi.

Source : enquête Conditions de travail 2013 (Dares).

\section{Des temps de travail contraints par l'activité}

La disponibilité temporelle observée pour les femmes non salariées est liée aux activités effectuées. Ce n'est pas le statut juridique qui impose d'être ainsi disponible. C'est bien, à l'inverse, parce que certaines activités induisent une forte disponibilité temporelle (dans leur organisation et structuration habituelles) que les emplois qui en relèvent s'inscrivent dans une logique non salariale. Le travail non salarié répondrait ainsi, parfois tout du moins, à la difficulté de rémunérer pleinement certaines formes de disponibilité temporelle au travail. C'est typiquement le cas pour les horaires décalés par rapport aux rythmes habituels et collectifs de travail, ou plus encore, pour les « temps creux » d'une activité agricole ou commerciale. Ces temps de travail impliquent d'être disponible sans pour autant que le travail soit « effectif » : il peut s'agir par exemple 
du temps d'attente d'un client ou de conditions météorologiques permettant la reprise d'activité. Ces périodes peuvent alors se rapprocher des astreintes connues par certaines catégories de salarié·e·s qui, si elles étaient considérées comme temps de travail, impliqueraient des durées plus longues mais des rémunérations horaires moyennes plus faibles. C'est du moins une interprétation possible des résultats précédents qui font ressortir deux contraintes touchant de manière plus intense les non-salariées, comme nous allons le voir : la première concerne davantage les plus diplômées et renvoie aux difficultés d'articulation des temps personnels et professionnels ; la seconde s'applique au contraire principalement aux non-diplômées et correspond à la faiblesse de leurs rémunérations, qui sont fréquemment en deçà du salaire minimum dès lors qu'on adopte une référence horaire.

\section{Liberté de temps de travail et difficultés d'articulation des temps : éléments de discussion}

L'analyse du temps de travail des femmes non salariées permet d'abord de revenir sur l'hypothèse de meilleures possibilités de conciliation entre vie personnelle et vie professionnelle pour les travailleuses qui sont à leur compte. Le constat a quelque chose de paradoxal : alors que les non-salariées déclarent disposer d'une réelle liberté pour déterminer leurs temps de travail, leur durée de travail est à la fois bien supérieure et plus étendue que celle des salariées.

Ce paradoxe se retrouve notamment lorsque l'on s'intéresse à l'effet de la situation familiale sur les temps de travail. D'une part, les non-salariées limitent davantage leur durée de travail quand elles sont en couple (par rapport aux salariées, cf. tableau 7, comme par rapport aux hommes non salariés, estimation non reproduite), ce qui conforte l'hypothèse d'une conciliation possible pour les femmes non salariées par

TABLEAU 7 - Durée hebdomadaire de travail et difficultés de conciliation des salariées et des non-salariées selon leur niveau de diplôme et leur situation familiale

\begin{tabular}{|c|c|c|c|c|}
\hline & \multicolumn{2}{|c|}{ Durée hebdomadaire } & \multicolumn{2}{|c|}{$\begin{array}{c}\text { Proportion déclarant des difficultés } \\
\text { de conciliation }(\%)\end{array}$} \\
\hline & Non-salariées & Salariées & Non-salariées & Salariées \\
\hline \multicolumn{5}{|l|}{ Niveau de qualification } \\
\hline Diplôme inférieur au bac & 41 h 31 & $31 \mathrm{~h} 12$ & 17,0 & 16,0 \\
\hline Bac à bac + 2 & 37 h 55 & 33 h 43 & 21,0 & 17,0 \\
\hline Supérieur à bac + 2 & 43 h 42 & 37 h 03 & 31,0 & 18,5 \\
\hline \multicolumn{5}{|l|}{ Situation familiale } \\
\hline Personne seule & 44 h 40 & 35 h 15 & 26,0 & 15,5 \\
\hline Couple sans enfant & 39 h 35 & $34 \mathrm{~h} 10$ & 17,5 & 13,5 \\
\hline Couple avec enfant & 40 h 25 & 33 h 20 & 22,5 & 18,5 \\
\hline Ensemble & 41 h 20 & 33 h 45 & 24,0 & 16,5 \\
\hline
\end{tabular}


rapport aux salariées. Mais, parallèlement, le profil de la journée de travail des femmes est bien moins affecté par leur situation familiale que par leur statut d'activité (graphique 6) : les heures de fin de journée des non-salariées ayant des enfants s'écartent finalement assez peu de celles qui vivent seules.

De même, les femmes non salariées déclarent des difficultés de conciliation des temps plus importantes, quels que soient leur niveau de diplôme et leur situation familiale. L'écart est toutefois particulièrement conséquent pour les plus diplômées : $31 \%$ des non-salariées détenant un diplôme supérieur à bac +2 déclarent ainsi ce type de difficultés, contre $21 \%$ des salariées ayant un niveau de diplôme équivalent. Il est en revanche plus surprenant que les conflits de ce type, déclarés par les nondiplômées, demeurent assez faibles (17 \% chez les non-salariées et $16 \%$ chez les salariées) au regard des nombreuses contraintes horaires qu'elles subissent. Une « sousdéclaration » n'est pas à exclure : comme pour de nombreuses variables subjectives, les travailleur·euse-s non salarié.e.es moins qualifié.e.s peuvent avoir tendance à nier une partie des difficultés rencontrées, à la fois dans une logique de rationalisation de

GRAPHIQUE 6 - Présence des salariées et des non-salariées sur les heures de fin de journée, selon leur situation familiale

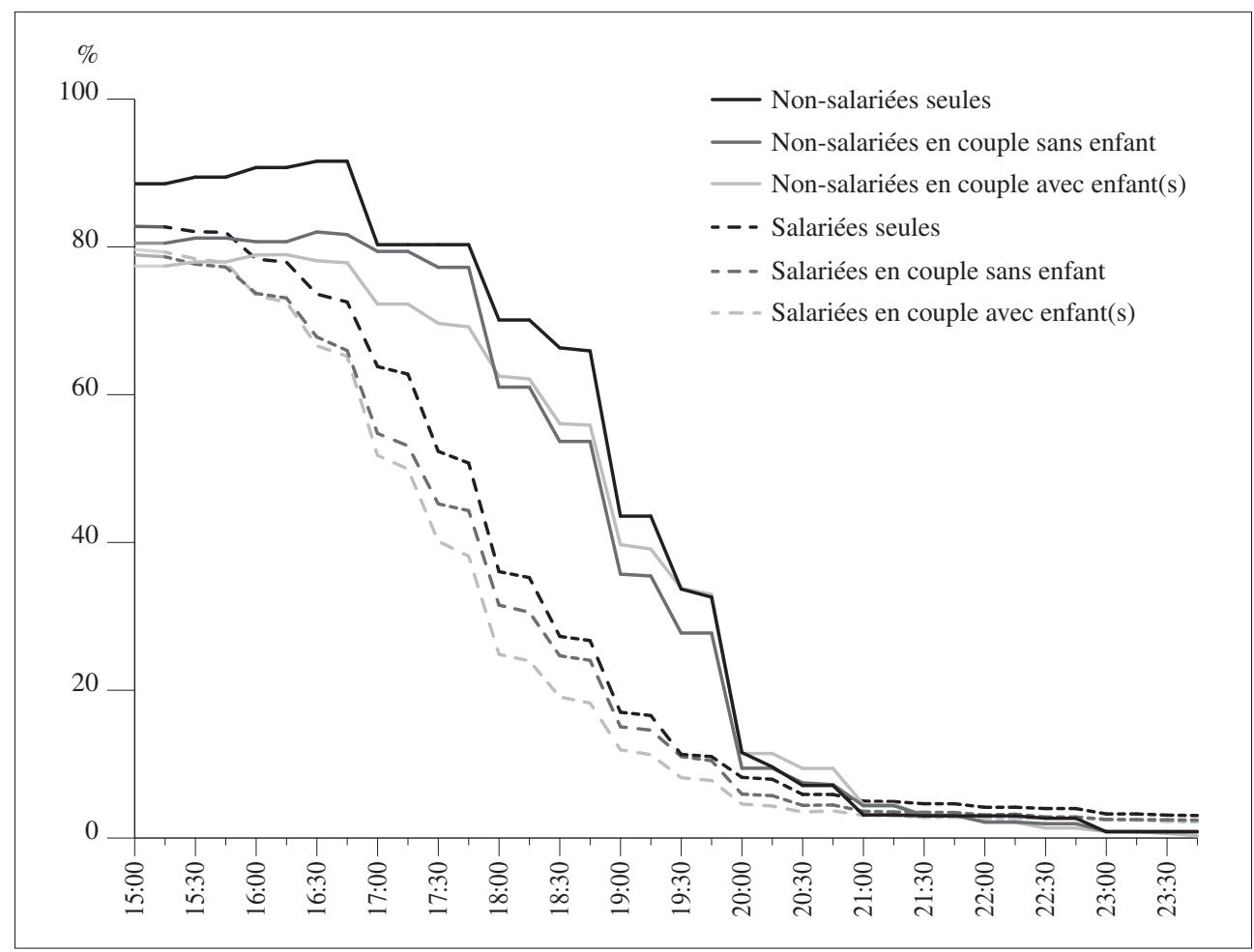

Lecture : en 2013, 90,7 \% des femmes non salariées vivant seules déclaraient travailler habituellement à 16 h 00.

Champ : ensemble des travailleuses en emploi.

Source : enquête Conditions de travail 2013 (Dares). 
difficultés qu'elles ou ils considèrent comme indépassables, mais aussi parce qu'elles ou ils valorisent l'autonomie, même relative, qu'elles ou ils ont en étant à leur compte, notamment lorsqu'elles ou ils ont auparavant exercé des emplois salariés d'exécution (TRotzier, 2005 ; Crasset, 2013).

Même sans intégrer ce possible effet, la liberté de temps de travail dont bénéficient juridiquement les travailleuses à leur compte semble en partie formelle au sens où elle est associée à de plus fortes expositions à des horaires longs, décalés ou imprévisibles. En schématisant, il est possible d'affirmer que les travailleur.euse-s préfèrent ne pas travailler en horaires longs ou décalés, mais que plus elles et ils ont le choix de leurs horaires, plus elles et ils travaillent en horaires longs ou décalés... La situation des non-salarié.e.s est de ce point de vue assez emblématique.

En économie, les travaux relatifs aux longues durées de travail peuvent suggérer plusieurs hypothèses complémentaires pour expliquer le décalage observé entre les horaires souhaités et ceux effectivement et, au moins en partie, librement consentis (HIRSCHMAN, 1984). Cette « discipline volontaire » s'expliquerait d'abord par des contraintes financières accrues ou plus explicites pour les non-salarié-e.s, liées par exemple à des investissements initiaux importants. Mais aussi par des formes de « courses » à la croissance des revenus influencées par des mécanismes de comparaison ou de recherches de consommations positionnelles (EASTMAn, 1998 ; GeOrges, 2001 ; Hamermesh, Slemrod, 2005 ; SCHOR, 2005). Le fait que les non-salarié.e-s qui travaillent toujours le dimanche ou le samedi appartiennent à des ménages dont les revenus sont plus faibles que les autres non salarié.e.s, alors que cet effet n'est pas ou peu visible chez les salarié·e.s, alimente cette hypothèse ${ }^{8}$. Un emploi peu rémunérateur rend nécessaire une extension des durées travaillées pour les non-qualifié.e.s tandis qu'une forme de « dépendance au surtravail » s'observerait chez les plus qualifiéee-s (HAMERMESH, SLEMROD, 2008).

L'engagement « excessif » dans l'activité professionnelle peut en effet également être lié à la signification sociale de l'emploi, qui est un élément essentiel de la position sociale. Un temps de travail long peut devenir le symbole d'un statut social enviable. Le « manque de temps » ou le « fait d'être débordé » serait un attribut social marquant la valeur de la personne (GERSHUNY, 2005). L'attitude des non-salarié.e.s à l'égard de leurs propres arrêts ou maladies étaierait cette hypothèse (SAUZE et al., 2011). Les temps de travail longs peuvent revêtir une dimension « ostentatoire » et le "présentéisme » être un comportement professionnel valorisé. Il est même parfois partie intégrante de l'identité des non-salarié.e·s, à l'image de ce que l'on observe parfois pour les salarié.e.s qualifié.e.s qui se fixent eux·elles-mêmes, au moins partiellement, leurs propres normes (BOUFFARTIGUE, 2012). Des travaux complémentaires, qui prendraient notamment appui sur des entretiens qualitatifs, seraient nécessaires pour éclaircir ces constats paradoxaux et éprouver les hypothèses avancées. Il n'en demeure pas moins que les femmes non salariées suivent le modèle d'activité masculin impliquant des

8. Le revenu mensuel des ménages de non-salarié·ess travaillant toujours le dimanche est de 3234 euros contre 4475 euros pour ceux elles ne travaillant jamais le dimanche (respectivement 3218 euros et 3434 euros pour les salarié·e·s). 
temps de travail importants et une présence régulière durant des périodes de travail atypiques. Globalement, le travail indépendant ne constitue ainsi pas un moyen de réduire ou d'assouplir le temps de travail mais représente plutôt une tension supplémentaire dans la gestion des temps : on observe bien à la fois une possibilité de s'adapter en cas d'urgences familiales et en même temps des horaires toujours plus tardifs en fin de journée. Ce constat explique en partie, et s'explique en retour par, le recours plus fréquent des femmes non salariées (et des ménages où travaille un·e non salarié.e en général) aux services domestiques rémunérés (МАRBOT, 2009). L'accroissement de l'activité professionnelle se traduit ici par une spécialisation plus forte et une externalisation plus fréquente de certaines tâches domestiques.

L'étude des journées de travail des non-salariées apporte de plus des éléments qui vont au-delà de la situation de cette seule catégorie de travailleuses. En effet, les caractéristiques de leur temps de travail apparaissent parfois comme une forme idéaltypique d'une liberté valorisée dans de nombreux discours portés par des responsables politiques ou des chercheur.euse-s en sciences sociales, sociologues (VIARD, 2006 ; JACQUOT, SETTI, 2006) ou économistes ${ }^{9}$. Or cette autonomie semble avoir des effets ambivalents aux niveaux individuel et social : si, lorsque l'autonomie augmente, le bien-être déclaré par les individus semble croître, les difficultés de conciliation et de synchronisation se révèlent également plus intenses. Qui plus est, les inégalités entre femmes et hommes, notamment lorsqu'elles ou ils ont des enfants à charge, sont sensiblement plus importantes chez les non-salarié.e.s. Des travaux exploratoires réalisés sur l'enquête Emploi du temps 2009-2010 indiquent que les ménages où l'homme est à son compte s'organisent de manière particulièrement inégalitaire (en ce qui concerne la répartition du travail domestique et parental, et cela en dépit d'un recours accru à des services externalisés pour l'entretien du domicile). Ces inégalités se retrouvent dans la participation gratuite plus fréquente des femmes de non-salariés à l'activité de leur conjoint : elle est bien supérieure à la situation symétrique (des hommes aidant leur conjointe non salariée ; cf. DELPHY, 2013).

\section{Les femmes non qualifiées : une « indépendance » économiquement très contrainte et aux frontières floues}

Si les difficultés d'articulation des temps sont principalement identifiées chez les non-salariées les plus qualifiées, la seconde difficulté à laquelle est confrontée une forte proportion de non-salariées touche au contraire prioritairement les non-qualifiées. Leur niveau de rémunération horaire, est ainsi particulièrement bas et peut même ne pas excéder le seuil de pauvreté lorsqu'il est ramené au niveau mensuel.

Le statut non salarié n'est pas associé à des rémunérations plus élevées pour toutes les catégories de travailleur.euse-s (tableau 8). Chez les femmes, seules les plus diplômées voient leur rémunération mensuelle être très légèrement supérieure à

9. Godet M. (2006), «L'erreur collective des 35 heures », Le Monde, 7 février. 
celle de leurs homologues salariées, tandis que pour les femmes moyennement et peu diplômées (comme pour les hommes non diplômés d'ailleurs), le statut de non-salarié est associé à des rémunérations en moyenne bien plus faibles ${ }^{10}$.

Ces effets sont bien plus prononcés pour les salaires horaires. Certes, les écarts se réduisent légèrement lorsque l'on intègre l'ensemble des revenus du ménage mais le constat demeure. Ainsi, le taux de pauvreté ${ }^{11}$ est particulièrement élevé pour les femmes non salariées peu ou pas diplômées : plus du tiers vit ainsi avec un revenu inférieur au seuil de pauvreté (tableau 9) ; une proportion importante d'entre elles travaillent dans le secteur agricole ou du petit commerce.

Cette situation très dégradée peut toutefois être contrebalancée par d'autres dimensions liées aux conditions de travail (intensité, autonomie par exemple) ou encore par les perspectives d'apprentissage sur le lieu de travail.

En effet, seules $12 \%$ des non-salariées non diplômées déclarent devoir constamment se dépêcher contre près de $20 \%$ des salariées de niveau de diplôme équivalent. De même, elles sont $35 \%$ à pouvoir faire varier les délais (soit un niveau équivalent aux femmes salariées ou à leur compte plus diplômées), contre seulement 17 \% des salariées non diplômées. L'intensité du travail semble enfin nettement plus faible pour les non-salariées, particulièrement dans la catégorie de faible niveau de qualification. L'hypothèse d'un travail « extensif », où les « creux » sont intégrés dans la journée de travail semble ainsi confirmée. Ce travail extensif serait le pendant, pour les non-salariées, des horaires fragmentés ou morcelés des salarié.e.s ayant un faible niveau de qualification (DEVETTER, 2016).

Les perceptions subjectives de la qualité de l'emploi (score de bien-être de l'Organisation mondiale de la santé, sentiment d'être exploitéee, qualité des relations avec les autres acteur.rice-s, etc.) sont également sensiblement meilleures pour les femmes à leur compte (que pour les salariées). Il est difficile d'interpréter ces résultats de façon causale, car des mécanismes d'auto-sélection ou du type « effet du travailleur sain » sont probablement à l'œuvre (Frigul, 2010 ; TROTZIER, 2005). Il reste que les non-salariées semblent accorder une valeur supérieure à leur emploi que les salariées.

Ces résultats apportent des éléments originaux concernant l'attractivité du statut non salarié. Pour les hommes diplômés, ce statut se traduit à la fois par des temps extensifs de travail et par une rémunération sensiblement plus élevée. Pour les femmes, la situation est quelque peu différente : leurs temps de travail sont également plus longs et plus contraints que ceux de leurs consœurs salariées, mais le gain de rémunération n'est pas assuré, il est même nettement négatif pour les non-salariées les moins diplômées. La situation de cette catégorie apparaît ainsi particulièrement dégradée, cumulant faibles rémunérations et disponibilité élevée.

10. La situation est toutefois moins désavantageuse pour les hommes que pour les femmes non salarié·e·s : ce statut s'accompagne pour eux de rémunérations mensuelles toujours supérieures, quel que soit leur niveau de diplôme.

11. Calculé ici comme le pourcentage de travailleur·euse·s appartenant à un ménage dont le revenu par unité de consommation est inférieur à 1000 euros en 2013, soit $60 \%$ du niveau de vie médian. 
TABLEAU 8 - Rémunérations des salarié·e•s et des non-salarié•e·s selon leur niveau de diplôme

En euros

\begin{tabular}{|c|c|c|c|c|}
\hline & Moyenne & Premier quartile & Médiane & Troisième quartile \\
\hline \multicolumn{5}{|l|}{ Femmes non salariées } \\
\hline Inférieur au bac & 1191 & 600 & 1000 & 1607 \\
\hline Bac à bac + 2 & 1458 & 417 & 961 & 2000 \\
\hline Supérieur à bac + 2 & 2400 & 833 & 2000 & 3333 \\
\hline \multicolumn{5}{|l|}{ Femmes salariées } \\
\hline Inférieur au bac & 1222 & 800 & 1200 & 1500 \\
\hline Bac à bac + 2 & 1510 & 1100 & 1400 & 1800 \\
\hline Supérieur à bac + 2 & 2184 & 1500 & 1907 & 2500 \\
\hline \multicolumn{5}{|l|}{ Hommes non salariés } \\
\hline Inférieur au bac & 1969 & 583 & 1250 & 2163 \\
\hline Bac à bac + 2 & 3076 & 833 & 1583 & 3000 \\
\hline Supérieur à bac + 2 & 3994 & 1250 & 2917 & 5000 \\
\hline \multicolumn{5}{|l|}{ Hommes salariés } \\
\hline Inférieur au bac & 1693 & 1300 & 1500 & 1900 \\
\hline Bac à bac + 2 & 2095 & 1400 & 1800 & 2500 \\
\hline Supérieur à bac + 2 & 3131 & 1900 & 2600 & 3700 \\
\hline
\end{tabular}

Lecture : les femmes non salariées ayant un niveau de diplôme inférieur au bac gagnent en moyenne 1191 euros par mois.

Champ : ensemble des travailleur-euse-s en emploi.

Source : enquête Conditions de travail 2013 (Dares).

TABLEAU 9 - Taux de pauvreté des ménages de salarié·e·s et de non-salarié•e·s selon leur niveau de diplôme

\begin{tabular}{|c|c|c|c|}
\hline & & Non-salarié·e·s & Salarié•e·s \\
\hline \multirow{3}{*}{ Femmes } & Inférieur au bac & 36 & 20 \\
\hline & Bac à bac + 2 & 23 & 10 \\
\hline & Supérieur à bac + 2 & 9 & 4 \\
\hline \multirow{3}{*}{ Hommes } & Inférieur au bac & 24 & 16 \\
\hline & $B a c$ à bac +2 & 13 & 7 \\
\hline & Supérieur à bac + 2 & 5 & 3 \\
\hline
\end{tabular}

Lecture : $36 \%$ des femmes non salariées ayant un niveau de diplôme inférieur au bac appartiennent à un ménage pauvre.

Champ : ensemble des travailleur.euse-s en emploi.

Source : enquête Conditions de travail 2013 (Dares).

Ce constat renvoie à la question plus large de la reconnaissance du travail féminin considéré comme peu qualifié. En effet, les activités concernées (petite production agricole, petit commerce, artisanat de service peu qualifié, etc.) peinent à déboucher sur des rémunérations supérieures au Smic (salaire minimum interprofessionnel de croissance). Alors que des mécanismes de calcul du temps de travail permettent de garantir un niveau minimal de rémunération pour certaines professions salariées (assistante maternelle, aide à domicile, employée de maison, nettoyeuse, femme de chambre par exemple), se mettre à son compte peut permettre l'exercice d'activités (agricultrice, commerçante, patronne de café ou coiffeuse) qui ne seraient pas rentables 
si elles étaient salariées et qui comprennent une part de travail gratuit réalisé dans ou à la frontière de la sphère domestique (DELPHY, 1983 ; NiCOURT, 2010). Les liens entre ces formes précarisées d'emploi salarié et la situation dégradée de certaines travailleuses non salariées ont été soulignés tant en France (ABDELNOUR, 2014) qu'au niveau international, notamment autour d'activités à connotation «domestique » (Selim, 2010). Certains pays comme l'Argentine ou le Brésil (George, Tizziani, 2016), promeuvent explicitement l'emploi indépendant pour réguler (de manière très légère) les « travailleurs domestiques $»^{12}$. Des phénomènes comparables ont été décrits pour des emplois masculins dans le secteur de la construction (Nicolas JounIN [2008] souligne ainsi une certaine perméabilité entre les statuts d'artisans et d'intérimaires) ou dans la sylviculture (GROS, 2015).

Les constats établis pour les non-salariées peu diplômées mettent en exergue la question de la « valorisation » de certaines activités, et notamment du travail féminin considéré comme non qualifié. L'institutionnalisation de ces emplois comme «non salariés » (ou à la marge du salariat, via des dispositifs comme le Chèque emploi service universel [Cesu]) permet ainsi un contournement du Smic et des protections liées à l'emploi et se traduit par des taux de pauvreté élevés ${ }^{13}$. À ce titre, ces logiques semblent fortement congruentes avec les évolutions récentes liées au développement des plates-formes numériques mobilisant des travailleur-euse-s indépendant·e.s précarisé·e·s (transport de personnes, livraison de repas, également de nombreux services à la personne).

En effet, les frontières entre travail domestique, travail salarié et non salarié sont parfois fragiles. Pour certaines activités féminisées, ces problématiques ne sont pas récentes. La situation des employées de maison, des femmes de ménages, des aides à domicile ou des assistantes maternelles est ambiguë : salariées dans leur immense majorité (le développement très récent de l'auto-entreprenariat dans ces activités n'est cependant pas un hasard), elles partagent de nombreux traits avec les travailleuses non salariées (DEVETTER, 2012). Tout d'abord leur temps de travail est fortement variable, et dépend directement de la « demande » des clients-employeurs, tandis que leur rémunération fluctue directement en fonction du marché des services aux particuliers. Ensuite, l'exercice de ces professions suppose la mobilisation de ressources personnelles importantes (logement, véhicule personnel, aides familiales, etc.). Enfin, ces métiers sont tout à la fois marqués par une grande autonomie et par un isolement

12. Ce n'est d'ailleurs pas un hasard si l'Organisation internationale du travail dans le numéro 68 de sa revue Travail publie un article sur les « Les mères entrepreneures au Québec : concilier travail et vie familiale » dans le dossier intitulé «Travail décent pour les travailleurs domestiques »; en ligne : http://www.ilo.org/wcmsp5/groups/public/@ dgreports/@dcomm/documents/publication/wcms_141263.pdf, consulté le 29 septembre 2017.

13. Situation que l'on retrouve dans bien d'autres pays comme en Italie (voir par exemple RUSPINI, SARACENO, 1999). 
important et l'absence des protections traditionnellement associées au statut salarié (institutions représentatives du personnel et avantages sociaux notamment).

Ainsi, la situation des travailleuses peu diplômées est parfois assez proche qu'elles soient salariées ou non salariées. Ces dernières se retrouvent également dans une situation souvent précaire : la disponibilité temporelle offerte est élevée, avec des amplitudes quotidiennes longues et de nombreux temps de travail atypiques (weekends et soirs par exemple), mais elle ne débouche que sur des rémunérations mensuelles et, plus encore, horaires faibles. Le statut de non-salariée de ces femmes semble alors plus une contrainte que la réelle valorisation de leur autonomie.

\section{BibliograPhiE}

ABDELNOUR S. (2014), «L'auto-entrepreneuriat : une gestion individuelle du sous-emploi », Nouvelle Revue du travail [en ligne], $\mathrm{n}^{\circ} 5:$ http://nrt.revues.org/1879, consulté le 13 octobre 2017.

Amossé T., Goux D. (2004), "Entries and Exits from Self-Employment in France over the Last Twenty Years", in Arum R., Muller W., The Reemergence of Self-Employment, Princeton/ Oxford, Princeton University Press, pp. 75-103.

ANDRIA (D') A. (2014), « Un éclairage sur le processus entrepreneurial des mampreneurs. Étude exploratoire de leur dynamique effectuale », Revue de l'entrepreneuriat, vol. 13, $\mathrm{n}^{\circ} 1$, pp. 11-33.

Arum R., Muller W. (2004), The Reemergence of Self-Employment, Princeton/Oxford, Princeton University Press.

BEFFy M. (2006), « Moins d'artisans, des professions libérales en plein essor », in Insee, France, portrait social. Édition 2006, Paris, Insee, coll. « Insee Références », pp. 139-156.

Bотеz L. (2010), «Les mères entrepreneures au Québec : concilier travail et vie familiale », Travail, $\mathrm{n}^{\circ} 68$, pp. 22-23.

Bouffartigue P. (2012), Temps de travail et temps de vie : les nouveaux visages de la disponibilité temporelle, Paris, Presses universitaires de France.

BURRI S. (2010), « La conciliation de la vie professionnelle, privée et familiale. L'approche juridique de l'Union européenne », Revue de l'OFCE, n 114, pp. 213-236.

Carrier C., Julien P.-A., Menvielle W. (2006), «Un regard critique sur l'entrepreneuriat féminin : une synthèse des études des 25 dernières années », Gestion, vol. 31, n 2, pp. 36-50.

CÉLÉRIER S. (dir.) (2014), Le Travail indépendant. Statut, activités et santé, Rueil-Malmaison, Éditions Liaisons.

CONSEIL D'ORIENTATION POUR L'EMPLOI (COE) (2014), L'Évolution des formes d'emploi. Rapport, 8 avril 2014, Paris, COE.

CRAsset O. (2013), «"On se fait mal un peu tous les jours", l'effet travailleur sain chez les maréchaux-ferrants », Travail et Emploi, $\mathrm{n}^{\circ} 136$, pp. 5-20. 
DelPhy C. (1983), «Agriculture et travail domestique : la réponse de la bergère à Engels », Nouvelles Questions féministes, $\mathrm{n}^{\circ}$ 5, pp. 3-17.

DelPhy C. (2013), L’Ennemi principal. 1, Économie politique du patriarcat, $3^{\mathrm{e}}$ éd., Paris, Syllepse.

Desrosières A, Thévenot L. (2002), Les Catégories socioprofessionnelles, $5^{\mathrm{e}}$ éd., Paris, La Découverte.

Devetter F.-X. (2001), Socioéconomie de la disponibilité temporelle au travail, thèse de sciences économiques, Université de Lille 1.

DevetTeR F.-X. (2002), «La régulation des temps de travail atypiques : entre allocation hiérarchique et transaction », Travail et Emploi, n ${ }^{\circ}$ 92, pp. 57-69.

Devetter F.-X. (2012), « Qualité de l'emploi et des conditions de travail des professionnelles de la petite enfance », Politiques sociales et familiales, $\mathrm{n}^{0} 109$, pp. 9-22.

DevetTer F.-X. (2016), “Cleaners Working Time: A Way to Avoid Minimum Wage Legislation”, $37^{\text {th }}$ Annual Conference of the International Working Party on Labour Market Segmentation, 6-8 July, Barcelona.

DuchÉNEAUt B., Orhan M. (2000), Les Femmes entrepreneurs en France, Paris, Éditions Seli Arslan.

Eastman C. (1998), "Working for Position: Women, Men and Managerial Work Hours", Industrial Relations, vol. $37, \mathrm{n}^{\circ} 1$, pp. 51-65.

EsPing Andersen G. (1999), Les Trois Mondes de l'État-providence, Paris, Presses universitaires de France.

EvaIn F., AMAR M. (2006), « Les indépendants », Insee première, nº 1084.

FAVRE F. (2009), « Hommes-femmes, des différences de revenu sensibles pour les nonsalariés », in Insee, Les Revenus d'activité des indépendants. Édition 2009, Paris, Insee, coll. «Insee références », pp. 31-45.

Fouquet A. (2005), «Les femmes chefs d'entreprise : le cas français », Travail, genre et sociétés, $\mathrm{n}^{\mathrm{o}} 13$, pp. 31-50.

FRIGUL N. (2010), « Les effets dissimulés du travail sur la santé de populations au chômage : le cas des contrats aidés dans le dispositif du RMI », Politix, nº 91, pp. 133-156.

George I., Tizziani A. (2016), «Entre le travail et l'assistance. Des dispositifs de "formalisation" du travail du care en Amérique latine en question (Argentine, Brésil) », Journées de l'AES, septembre 2016, Lille.

GeOrges D. (2001), "Driven to Spend: Longer Work Hours as a Byproduct of Market Forces", in Golden L., Figart D. M. (eds), Working Time. International Trends, Theory and Policy Perspectives, London, Routledge, pp. 127-143.

Gershuny J. (2005), "Busyness as the Badge of Honor for the New Superordinate Working Class", Social Research, vol. 72, n 2, pp. 287-314. 
Gros J. (2015), Des classes populaires à la lisière du salariat. Une analyse des bûcherons entre emploi, marché et stratification sociale, thèse de doctorat en sociologie, Paris, École des hautes études en sciences sociales.

Guggemos F., VidAlenc J. (2014), « Une photographie du marché du travail en 2013 », Insee première, $\mathrm{n}^{\circ} 1516$.

HAmermesh D. S., Slemrod J. (2005), “The Economics of Workaholism: We Should Not Have Worked on This Paper", NBER Working Paper, n 11566.

Hamermesh D. S., Slemrod J. (2008), "The Economics of Workaholism: We Should Not Have Worked on This Paper", The B.E. Journal of Economic Analysis \& Policy [en ligne], vol. 8, n ${ }^{\circ} 1$ : https://doi.org/10.2202/1935-1682.1793, consulté le 17 octobre 2017.

Hirschman A. (1984), L'Économie comme science morale et politique, Paris, Gallimard, Seuil. INSEE (2004), « Les femmes créatrices d'entreprises », Insee Résultats, série «Économie », $\mathrm{n}^{\mathrm{o}} 16$, août.

InSERM (InSTITUT NATIONAL DE LA SANTÉ ET DE LA RECHERCHE MÉDICALE) (2011), Stress au travail et santé : situation chez les indépendants. Synthèse et recommandations. Expertise collective, Paris, Inserm.

JACQUOT L., SETTi N. (2006), « Les ambivalences des 35 heures dans un organisme de sécurité sociale ou l'utopie du temps choisi », Temporalités [en ligne], $\mathrm{n}^{\circ} 4$ : https://temporalites.revues. org/324, consulté le 17 octobre 2017.

Jounin N. (2008), Chantier interdit au public. Enquête parmi les travailleurs du bâtiment, Paris, La Découverte.

LuRTon G., Toutlemonde F. (2007), « Les déterminants de l'emploi non salarié en France depuis 1970 », Document d'études, n 129, Paris, Dares.

MARвот C. (2009), « Le recours aux services à domicile et ses déterminants en France. Une analyse au cœur du ménage », Travail, genre et sociétés, $\mathrm{n}^{\circ} 22$, pp. 31-52.

Marchand O., ThÉLot C. (1997), Le Travail en France (1800-2000), Paris, Nathan.

Nel P., Maritz A., Thongprovati O. (2010), "Motherhood and Entrepreneurship: The Mumpreneur Phenomenon", The International Journal of Organizational Innovation, vol. 3, $\mathrm{n}^{\mathrm{o}} 1$, pp. 6-34.

Nicourt C. (2010), « Le lent dévoilement du travail des agricultrices », VertigO [en ligne], vol. 14, $\mathrm{n}^{\mathrm{o}} 1$ : https://vertigo.revues.org/14731, consulté le 17 octobre 2017.

Omalek L., Rioux L. (coord.) (2015), Emploi et revenus des indépendants, Paris, Insee, coll. «Insee références ».

RAPELLi S., LESPAGNOL C. (2007), «La population des travailleurs non salariés à l'horizon $2030 »$, Colloque « Prospective et entreprise », Paris, 6 décembre 2007.

Ruspini E, SARACENo C. (1999), «Précarité des revenus, pauvreté des salaires : le cas des femmes en Italie », Travail, genre et sociétés, $\mathrm{n}^{\mathrm{0}} 1$, pp. 87-118. 
SAlEMBiER L., ThÉRON G. (2016), « Revenus d'activité des non-salariés en 2014. Hausse pour les indépendants "classiques", baisse pour les auto-entrepreneurs », Insee première, n” 1627. Sauze L., Ha-Vinh P., RÉGnard P. (2011), «Affections de longue durée et différences de morbidité entre travailleurs salariés et travailleurs indépendants. Introduction », Pratiques et organisation des soins, vol. 42, $\mathrm{n}^{\circ} 1$, p. 1-9.

SCHOR J. B. (2005), "Sustainable Consumption and Worktime Reduction”, Journal of Industrial Ecology, vol. 9, n' 1-2, pp. 37-50.

Selim M. (2010), « La face cachée des femmes-outils », L'Homme et la Société, n ${ }^{\circ}$ 176-177, pp. 253-266.

TROTZIER T. (2005), «Vingt ans de trajectoire après un licenciement collectif. Ouvrières et ouvriers », Revue économique, vol. 56, n² 2, pp. 257-275.

VIARD J. (2006), Éloge de la mobilité. Essai sur le capital temps libre et la valeur travail, La Tour-d'Aigues, Éditions de l'Aube. 